\title{
THE CAUCHY PROBLEM ON THE COMPRESSIBLE TWO-FLUIDS EULER-MAXWELL EQUATIONS
}

\author{
RENJUN DUAN, QINGQING LIU, AND CHANGJIANG ZHU*
}

\begin{abstract}
In this paper, we are concerned with the Cauchy problem on the compressible isentropic two-fluids Euler-Maxwell equations in three dimensions. The global existence of solutions near constant steady states with the vanishing electromagnetic field is established, and also the time-decay rates of perturbed solutions in $L^{q}$ space for $2 \leq q \leq \infty$ are obtained. The proof for existence is due to the classical energy method, and the investigation of the large-time behavior is based on the linearized analysis of the one-fluid Euler-Maxwell equations and the damped Euler equations. As a byproduct of our approach, some timedecay rates obtained in [18] for the nonlinear damped Euler system are improved.
\end{abstract}

\section{Contents}

1. Introduction 1

2. Global solutions for the nonlinear system 4

2.1. Reformulation of the problem 4

2.2. A priori estimates 6

3. Linearized homogeneous system 11

3.1. Representation of solutions for (3.13) and (3.14). 14

3.2. Refined $L^{p}-L^{q}$ time-decay property 15

4. Decay in time for the non-linear system 18

4.1. Time rate for full instant energy functional $\quad 18$

4.2. Time rate for higher-order instant energy functional $\quad 21$

4.3. Time rate in $L^{q} \quad 23$

5. Appendix 26

References $\quad 30$

\section{INTRODUCTION}

The two-fluids Euler-Maxwell system in plasma physics describe dynamics of two separate compressible fluids of ions and electrons interacting with their self-consistent electromagnetic field [17]. Many famous nonlinear dispersive PDE, such as Zakharovs equation, nonlinear Schrödinger equations, as well as KdV equations, can be formally derived from two-fluids Euler-Maxwell system under various asymptotic limits, see [1, 2] and references therein. In this paper, we consider the compressible isentropic two-fluids Euler-Maxwell system in three

Date: October 7, 2011.

2000 Mathematics Subject Classification. 35Q35, 35P20.

Key words and phrases. Bipolar Euler-Maxwell System, plasma physics, Euler equation with damping, time-decay rate.

*Corresponding author. Email: cjzhu@mail.ccnu.edu.cn (C.J. Zhu). 
dimensions, taking the form of

$$
\left\{\begin{array}{l}
\partial_{t} n_{ \pm}+\nabla \cdot\left(n_{ \pm} u_{ \pm}\right)=0 \\
\partial_{t} u_{ \pm}+u_{ \pm} \cdot \nabla u_{ \pm}+\frac{1}{n_{ \pm}} \nabla p_{ \pm}\left(n_{ \pm}\right)=\mp\left(E+u_{ \pm} \times B\right)-\nu_{ \pm} u_{ \pm} \\
\partial_{t} E-\nabla \times B=n_{+} u_{+}-n_{-} u_{-}, \\
\partial_{t} B+\nabla \times E=0, \\
\nabla \cdot E=n_{-}-n_{+}, \quad \nabla \cdot B=0 .
\end{array}\right.
$$

Here, $n_{ \pm}=n_{ \pm}(t, x) \geq 0$ and $u_{ \pm}=u_{ \pm}(t, x) \in \mathbb{R}^{3}$ with $t>0, x \in \mathbb{R}^{3}$, respectively, are densities and velocities of the ion (-) and electron $(+) . E=E(t, x) \in \mathbb{R}^{3}$ and $B=B(t, x) \in \mathbb{R}^{3}$ denote the electromagnetic field. Initial data is given as

$$
\left.\left[n_{ \pm}, u_{ \pm}, E, B\right]\right|_{t=0}=\left[n_{ \pm 0}, u_{ \pm 0}, E_{0}, B_{0}\right], \quad x \in \mathbb{R}^{3},
$$

with the compatibility conditions

$$
\nabla \cdot E_{0}=n_{-0}-n_{+0}, \quad \nabla \cdot B_{0}=0, \quad x \in \mathbb{R}^{3} .
$$

The pressure functions $p_{ \pm}(\cdot)$ depending only on the density satisfy the power law $p_{ \pm}\left(n_{ \pm}\right)=$ $A_{ \pm} n_{ \pm}^{\gamma}$ with constants $A_{ \pm}>0$ and the adiabatic exponent $\gamma>1$. Constants $\nu_{ \pm}>0$ are the velocity relaxation frequency of ions and electrons. Notice that in general the Euler-Maxwell system depends on many other physical parameters; refer to [1]. Here, we have skipped them and only consider how the strictly positive relaxation frequency play in the whole theory. Moreover, through this paper, we assume $A_{+}=A_{-}=A$ and $\nu_{+}=\nu_{-}=\nu$, and for simplicity we also let $A=\nu=1$. In such simple case, as we shall show later on, the corresponding linearized Euler-Maxwell system can be written as two-decoupled subsystems, i.e., the onefluid Euler-Maxwell system and the damped Euler system, which is essentially used in the study of the time-decay property for the two-fluids Euler-Maxwell system above. The case for general choices of $A_{ \pm}$and $\nu_{ \pm}$that lead to more complex coupling structure is left for the future study.

Before stating the main result, let us recall some previous related work. For the one-fluid Euler-Maxwell system when ions only provide a uniform constant background, by using the fractional Godunov scheme as well as the compensated compactness argument, Chen-JeromeWang [4] proved global existence of weak solutions to the initial-boundary value problem in one space dimension for arbitrarily large initial data in $L^{\infty}$. Jerome [11] established a local smooth solution theory for the Cauchy problem over $\mathbb{R}^{3}$ by adapting the classical semigroupresolvent approach of Kato [13]. Peng-Wang [16] justified convergence of the compressible Euler-Maxwell system to the incompressible Euler system for well-prepared smooth initial data. Recently, Duan [6] proved the existence and uniqueness of global smooth solutions with small amplitude to the one-fluid Euler-Maxwell system in three space dimensions, and obtained the optimal large-time behavior of solutions in terms of the detailed analysis of the Green's function. The similar results are independently given by Ueda-Wang-Kawashima [22] and Ueda-Kawashima [21] by using the pure time-weighted energy method. Much more studies have been made for the Euler-Poisson system when the magnetic field is absent; see $[8,9,14,5,15,3]$ and references therein for discussion and analysis of the different issues such as the existence of global smooth irrotational flow [8] for an electron fluid and [9] for the ion dynamics, large time behavior of solutions [14], stability of star solutions [5, 15] and finite time blow-up [3].

However, there are few results on the two-fluids Euler-Maxwell system. As we pointed out before, depending on the choice of physical parameters, the case of two-fluids exhibits 
much more complex decay structure than that of one-fluid; see (3.1) as well as (3.4) and (3.6). The detailed analysis of the linearized system in the case when $A_{+}=A_{-}=1$ and $\nu_{+}=\nu_{-}=1$ will be given in Section 3, cf. Proposition 3.1 and Theorem 3.1. Concerning the nonlinear two-fluids Euler-Maxwell system, the main result is stated as follows. Notations will be explained at the end of this section.

Theorem 1.1. Let $N \geq 4$ and (1.3) hold. There are $\delta_{0}>0, C_{0}$ such that if

$$
\left\|\left[n_{ \pm 0}-1, u_{ \pm 0}, E_{0}, B_{0}\right]\right\|_{N} \leq \delta_{0},
$$

then, the Cauchy problem (1.1)-(1.2) of the Euler-Maxwell system admits a unique global solution $\left[n_{ \pm}(t, x), u_{ \pm}(t, x), E(t, x), B(t, x)\right]$ with

$$
\left[n_{ \pm}(t, x)-1, u_{ \pm}(t, x), E(t, x), B(t, x)\right] \in C\left([0, \infty) ; H^{N}\left(\mathbb{R}^{3}\right)\right) \cap \operatorname{Lip}\left([0, \infty) ; H^{N-1}\left(\mathbb{R}^{3}\right)\right),
$$

and

$$
\sup _{t \geq 0}\left\|\left[n_{ \pm}(t)-1, u_{ \pm}(t), E(t), B(t)\right]\right\|_{N} \leq C_{0}\left\|\left[n_{ \pm 0}-1, u_{ \pm 0}, E_{0}, B_{0}\right]\right\|_{N} .
$$

Moreover, there are $\delta_{1}>0, C_{1}$ such that if

$$
\left\|\left[n_{ \pm 0}-1, u_{ \pm 0}, E_{0}, B_{0}\right]\right\|_{13}+\left\|\left[n_{ \pm 0}-1, u_{ \pm 0}, E_{0}, B_{0}\right]\right\|_{L^{1}} \leq \delta_{1},
$$

then, the solution $\left[n_{ \pm}(t, x), u_{ \pm}(t, x), E(t, x), B(t, x)\right]$ satisfies that for any $t \geq 0$,

$$
\begin{aligned}
& \left\|n_{+}(t)-n_{-}(t)\right\|_{L^{q}} \leq C_{1}(1+t)^{-2-\frac{1}{q}} \\
& \left\|n_{+}(t)+n_{-}(t)-2\right\|_{L^{q}} \leq C_{1}(1+t)^{-\frac{3}{2}+\frac{3}{2 q}} \\
& \|\left[u_{+}(t) \pm u_{-}(t), E(t) \|_{L^{q}} \leq C_{1}(1+t)^{-2+\frac{3}{2 q}}\right. \\
& \|B(t)\|_{L^{q}} \leq C_{1}(1+t)^{-\frac{3}{2}+\frac{3}{2 q}}
\end{aligned}
$$

with $2 \leq q \leq \infty$.

The proof of existence in Theorem 1.1 above is based on the classical energy method. As in [6], the key point is to obtain the uniform-in-time a priori estimates in the form of

$$
\mathcal{E}_{N}(V(t))+\lambda \int_{0}^{t} \mathcal{D}_{N}(V(s)) d s \leq \mathcal{E}_{N}\left(V_{0}\right)
$$

where $V(t)$ is the perturbation of solutions, and $\mathcal{E}_{N}(\cdot), \mathcal{D}_{N}(\cdot)$ denote the energy functional and energy dissipation rate functional. Although along the same line, our construction of $\mathcal{E}_{N}(\cdot)$ and $\mathcal{D}_{N}(\cdot)$ is a little different from that for the one-fluid case as in [6] because of the more complex structure of two-fluids system. In particular, since the system is degenerate over some components of the whole solution, one has to construct some interactive functionals so as to capture the optimal form of the energy dissipation rate which plays a vital role for the study of time-decay property of solutions to the nonlinear system. We here notice the general theory of hypocoercivity in [23].

Moreover, in order to obtain the rates of convergence of solutions in Theorem 1.1, our approach is the combination of the analysis of Green's function of the linearized system and the refined energy estimates with the help of the Duhamel's principle. Related to this approach, we only mention [12] for the systematic study of systems of a hyperbolic-parabolic composite type, and also notice that the theoretical framework developed in [12] can not be applied to the two-fluids Euler-Maxwell system considered here. Thus, we first discuss the time-decay rates of the linearized equations. When $\nu_{+}=\nu_{-}$, the linearized homogeneous equations of the two-fluids Euler-Maxwell system (1.1) can be written as two decoupled subsystems (3.10) and (3.13). For (3.10), the corresponding results can be obtained in a 
parallel way as [6]. It is observed that (3.13) is the linearized homogeneous system also corresponding to the 3-D compressible Euler equations with damping (5.1). For the Cauchy problem (5.1)-(5.2) of the damped Euler system, Sideris-Thomases-Wang [18] actually has obtained the time-decay rates of solutions by using the Fourier analysis. Here we shall revisit this issue to consider the optimal rates of each component in the solution by exploring the explicit solution to the Cauchy problem (3.13)-(3.14) in the Fourier space. In fact, our Proposition 5.1 in Appendix is an improved result of [18, Lemma 6.1] in the sense that the momentum component has an extra time-decay $(1+t)^{-1 / 2}$ in $L^{2}$. Notice that the timedecay rates obtained in Corollary 3.1 can be used to refine [18, Theorem 6.1]. In that sense, Appendix can be viewed as a generalization of [18].

Let us introduce some notations for the use throughout this paper. $C$ denotes some positive (generally large) constant and $\lambda$ denotes some positive (generally small) constant, where both $C$ and $\lambda$ may take different values in different places. For two quantities $a$ and $b, a \sim b$ means $\lambda a \leq b \leq \frac{1}{\lambda} a$ for a generic constant $0<\lambda<1$. For any integer $m \geq 0$, we use $H^{m}, \dot{H}^{m}$ to denote the usual Sobolev space $H^{m}\left(\mathbb{R}^{3}\right)$ and the corresponding $m$-order homogeneous Sobolev space, respectively. Set $L^{2}=H^{m}$ when $m=0$. For simplicity, the norm of $H^{m}$ is denoted by $\|\cdot\|_{m}$ with $\|\cdot\|=\|\cdot\|_{0}$. We use $\langle\cdot, \cdot\rangle$ to denote the inner product over the Hilbert space $L^{2}\left(\mathbb{R}^{3}\right)$, i.e. $\langle f, g\rangle=\int_{\mathbb{R}^{3}} f(x) g(x) d x, f=f(x), g=g(x) \in L^{2}\left(\mathbb{R}^{3}\right)$. For a multi-index $\alpha=\left[\alpha_{1}, \alpha_{2}, \alpha_{3}\right]$, we denote $\partial^{\alpha}=\partial_{x_{1}}^{\alpha_{1}} \partial_{x_{2}}^{\alpha_{2}} \partial_{x_{3}}^{\alpha_{3}}$. The length of $\alpha$ is $|\alpha|=\alpha_{1}+\alpha_{2}+\alpha_{3}$. For simplicity, we also set $\partial_{j}=\partial_{x_{j}}, j=1,2,3$.

We conclude this section by stating the arrangement of the rest of this paper. In Section 2, we reformulate the Cauchy problem under consideration and prove the global existence and uniqueness of solutions. In Section 3, we investigate the linearized homogeneous system to obtain the $L^{p}-L^{q}$ time-decay property and the explicit representation of solutions. In Section 4, we study the time-decay rates of solutions to the reformulated nonlinear system and finish the proof of Theorem 1.1. In the last Section 5, we use an appendix to investigate the time-decay rates of solutions to the Cauchy problem on the damped Euler system (5.1), which improves the corresponding results in [18].

\section{Global SOlutions for the NONLinear SYSTEM}

2.1. Reformulation of the problem. Let $\left[n_{ \pm}, u_{ \pm}, E, B\right]$ be a smooth solution to the Cauchy problem of the Euler-Maxwell system (1.1) with given initial data (1.2) satisfying (1.3). Set

$$
\left\{\begin{array}{l}
\sigma_{ \pm}(t, x)=\frac{2}{\gamma-1}\left\{\left[n_{ \pm}\left(\frac{t}{\sqrt{\gamma}}, x\right)\right]^{\frac{\gamma-1}{2}}-1\right\}, v_{ \pm}=\frac{1}{\sqrt{\gamma}} u_{ \pm}\left(\frac{t}{\sqrt{\gamma}}, x\right), \\
\tilde{E}=\frac{1}{\sqrt{\gamma}} E\left(\frac{t}{\sqrt{\gamma}}, x\right), \tilde{B}=\frac{1}{\sqrt{\gamma}} B\left(\frac{t}{\sqrt{\gamma}}, x\right) .
\end{array}\right.
$$


Then, $V:=\left[\sigma_{ \pm}, v_{ \pm}, \tilde{E}, \tilde{B}\right]$ satisfies

$$
\left\{\begin{array}{l}
\partial_{t} \sigma_{ \pm}+v_{ \pm} \cdot \nabla \sigma_{ \pm}+\left(\frac{\gamma-1}{2} \sigma_{ \pm}+1\right) \nabla \cdot v_{ \pm}=0 \\
\partial_{t} v_{ \pm}+\left(\frac{\gamma-1}{2} \sigma_{ \pm}+1\right) \nabla \sigma_{ \pm}+v_{ \pm} \cdot \nabla v_{ \pm}=\mp\left(\frac{1}{\sqrt{\gamma}} \tilde{E}+v_{ \pm} \times \tilde{B}\right)-\frac{1}{\sqrt{\gamma}} v_{ \pm}, \\
\partial_{t} \tilde{E}-\frac{1}{\sqrt{\gamma}} \nabla \times \tilde{B}=\frac{1}{\sqrt{\gamma}} v_{+}+\frac{1}{\sqrt{\gamma}}\left[\Phi\left(\sigma_{+}\right)+\sigma_{+}\right] v_{+}-\frac{1}{\sqrt{\gamma}} v_{-}-\frac{1}{\sqrt{\gamma}}\left[\Phi\left(\sigma_{-}\right)+\sigma_{-}\right] v_{-}, \\
\partial_{t} \tilde{B}+\frac{1}{\sqrt{\gamma}} \nabla \times \tilde{E}=0, \\
\nabla \cdot \tilde{E}=-\frac{1}{\sqrt{\gamma}}\left[\Phi\left(\sigma_{+}\right)+\sigma_{+}\right]+\frac{1}{\sqrt{\gamma}}\left[\Phi\left(\sigma_{-}\right)+\sigma_{-}\right], \quad \nabla \cdot \tilde{B}=0 . \quad t>0, x \in \mathbb{R}^{3},
\end{array}\right.
$$

with initial data

$$
\left.V\right|_{t=0}=V_{0}:=\left[\sigma_{ \pm 0}, v_{ \pm 0}, \tilde{E}_{0}, \tilde{B}_{0}\right], \quad x \in \mathbb{R}^{3} .
$$

Here, $\Phi(\cdot)$ is defined by

$$
\Phi(\sigma)=\left(\frac{\gamma-1}{2} \sigma+1\right)^{\frac{2}{\gamma-1}}-\sigma-1,
$$

and $V_{0}=\left[\sigma_{ \pm 0}, v_{ \pm 0}, \tilde{E}_{0}, \tilde{B}_{0}\right]$ is given from $\left[n_{ \pm 0}, u_{ \pm 0}, E_{0}, B_{0}\right]$ according to the transform (2.1), and hence $V_{0}$ satisfies

$$
\left\{\begin{array}{l}
\nabla \cdot \tilde{E}_{0}=-\frac{1}{\sqrt{\gamma}}\left[\Phi\left(\sigma_{+0}\right)+\sigma_{+0}\right]+\frac{1}{\sqrt{\gamma}}\left[\Phi\left(\sigma_{-0}\right)+\sigma_{-0}\right], \\
\nabla \cdot \tilde{B}_{0}=0, \quad x \in \mathbb{R}^{3} .
\end{array}\right.
$$

In what follows, the integer $N \geq 4$ is always assumed. Besides, for $V=\left[\sigma_{ \pm}, v_{ \pm}, \tilde{E}, \tilde{B}\right]$, we define the full instant energy functional $\mathcal{E}_{N}(V(t))$ and the high-order instant energy functional $\mathcal{E}_{N}^{h}(V(t))$ by

$$
\begin{aligned}
\mathcal{E}_{N}(V(t))= & \|V(t)\|_{N}^{2} \\
& +\kappa_{1} \sum_{|\alpha| \leq N-1}\left(\left\langle\partial^{\alpha} v_{+}, \nabla \partial^{\alpha} \sigma_{+}\right\rangle+\left\langle\partial^{\alpha} v_{-}, \nabla \partial^{\alpha} \sigma_{-}\right\rangle\right) \\
& +\kappa_{2} \sum_{|\alpha| \leq N-1}\left\langle\partial^{\alpha}\left(v_{+}-v_{-}\right), \nabla \partial^{\alpha} \tilde{E}\right\rangle-\kappa_{3} \sum_{|\alpha| \leq N-2}\left\langle\nabla \times \partial^{\alpha} \tilde{E}, \partial^{\alpha} \tilde{B}\right\rangle
\end{aligned}
$$

and

$$
\begin{aligned}
\mathcal{E}_{N}^{h}(V(t)) & =\|\nabla V(t)\|_{N-1}^{2}+\kappa_{1} \sum_{1 \leq|\alpha| \leq N-1}\left(\left\langle\partial^{\alpha} v_{+}, \nabla \partial^{\alpha} \sigma_{+}\right\rangle+\left\langle\partial^{\alpha} v_{-}, \nabla \partial^{\alpha} \sigma_{-}\right\rangle\right) \\
& +\kappa_{2} \sum_{1 \leq|\alpha| \leq N-1}\left\langle\partial^{\alpha}\left(v_{+}-v_{-}\right), \nabla \partial^{\alpha} \tilde{E}\right\rangle-\kappa_{3} \sum_{1 \leq|\alpha| \leq N-2}\left\langle\nabla \times \partial^{\alpha} \tilde{E}, \partial^{\alpha} \tilde{B}\right\rangle,
\end{aligned}
$$

respectively, where $0<\kappa_{3} \ll \kappa_{2} \ll \kappa_{1} \ll 1$ are constants to be properly chosen in the later proof. Notice that since all constants $\kappa_{i}(i=1,2,3)$ are small enough, one has

$$
\mathcal{E}_{N}(V(t)) \sim\left\|\left[\sigma_{ \pm}, v_{ \pm}, \tilde{E}, \tilde{B}\right]\right\|_{N}^{2}, \quad \mathcal{E}_{N}^{h}(V(t)) \sim\left\|\nabla\left[\sigma_{ \pm}, v_{ \pm}, \tilde{E}, \tilde{B}\right]\right\|_{N-1}^{2}
$$

We further define the dissipation rates $\mathcal{D}_{N}(V(t)), \mathcal{D}_{N}^{h}(V(t))$ by

$$
\begin{aligned}
\mathcal{D}_{N}(V(t))=\left\|\left[v_{+}, v_{-}\right]\right\|_{N}^{2}+ & \left\|\nabla\left[\sigma_{+}, \sigma_{-}\right]\right\|_{N-1}^{2} \\
& +\|\nabla[\tilde{E}, \tilde{B}]\|_{N-2}^{2}+\|\tilde{E}\|^{2}+\left\|\sigma_{+}-\sigma_{-}\right\|^{2},
\end{aligned}
$$




$$
\begin{aligned}
\mathcal{D}_{N}^{h}(V(t))=\| \nabla\left[v_{+}, v_{-}\right] & \left\|_{N-1}^{2}+\right\| \nabla^{2}\left[\sigma_{+}, \sigma_{-}\right] \|_{N-2}^{2} \\
& +\left\|\nabla^{2}[\tilde{E}, \tilde{B}]\right\|_{N-3}^{2}+\|\nabla \tilde{E}\|^{2}+\left\|\nabla\left(\sigma_{+}-\sigma_{-}\right)\right\|^{2} .
\end{aligned}
$$

Notice that different from the one-fluid case as in [6], $\left\|\sigma_{+}+\sigma_{-}\right\|^{2}$ and hence $\left\|\sigma_{ \pm}\right\|^{2}$ are excluded from $\mathcal{D}_{N}(V(t))$, and $\left\|\nabla\left(\sigma_{+}+\sigma_{-}\right)\right\|^{2}$ and thus $\left\|\nabla \sigma_{ \pm}\right\|^{2}$ do not appear in $\mathcal{D}_{N}^{h}(V(t))$ any more. Now, concerning the reformulated Cauchy problem (2.2)-(2.3), one has the following global existence result.

Proposition 2.1. Suppose (2.4) for given initial data $V_{0}=\left[\sigma_{ \pm 0}, v_{ \pm 0}, \tilde{E}_{0}, \tilde{B}_{0}\right]$. Then, there are $\mathcal{E}_{N}(\cdot)$ and $\mathcal{D}_{N}(\cdot)$ given by $(2.5)$ and (2.7) such that the following holds true. If $\mathcal{E}_{N}\left(V_{0}\right)>0$ is small enough, the Cauchy problem (2.2)-(2.3) admits a unique global nonzero solution $V=\left[\sigma_{ \pm}, v_{ \pm}, \tilde{E}, \tilde{B}\right]$ satisfying

$$
V \in C\left([0, \infty) ; H^{N}\left(\mathbb{R}^{3}\right)\right) \cap \operatorname{Lip}\left([0, \infty) ; H^{N-1}\left(\mathbb{R}^{3}\right)\right),
$$

and

$$
\mathcal{E}_{N}(V(t))+\lambda \int_{0}^{t} \mathcal{D}_{N}(V(s)) d s \leq \mathcal{E}_{N}\left(V_{0}\right)
$$

for any $t \geq 0$.

Moreover, solutions obtained in Proposition 2.1 indeed decay in time with some rates under some extra regularity and integrability conditions on initial data. For that, given $V_{0}=\left[\sigma_{ \pm 0}, v_{ \pm 0}, \tilde{E}_{0}, \tilde{B}_{0}\right]$, set $\epsilon_{m}\left(V_{0}\right)$ as

$$
\epsilon_{m}\left(V_{0}\right)=\left\|V_{0}\right\|_{m}+\left\|\left[\sigma_{ \pm 0}, v_{ \pm 0}, \tilde{E}_{0}, \tilde{B}_{0}\right]\right\|_{L^{1}},
$$

for the integer $m \geq 4$. Then one has

Proposition 2.2. Suppose (2.4) for given initial data $V_{0}=\left[\sigma_{ \pm 0}, v_{ \pm 0}, \tilde{E}_{0}, \tilde{B}_{0}\right]$. If $\epsilon_{N+2}\left(V_{0}\right)>$ 0 is small enough, then the solution $V=\left[\sigma_{ \pm}, v_{ \pm}, \tilde{E}, \tilde{B}\right]$ satisfies

$$
\|V(t)\|_{N} \leq C \epsilon_{N+2}\left(V_{0}\right)(1+t)^{-\frac{3}{4}}
$$

for any $t \geq 0$. Furthermore, if $\epsilon_{N+6}\left(V_{0}\right)>0$ is small enough, then the solution $V=$ $\left[\sigma_{ \pm}, v_{ \pm}, \tilde{E}, \tilde{B}\right]$ also satisfies

$$
\|\nabla V(t)\|_{N-1} \leq C \epsilon_{N+6}\left(V_{0}\right)(1+t)^{-\frac{5}{4}}
$$

for any $t \geq 0$.

The existence result in Theorem 1.1 directly follows from Proposition 2.1, and the derivation of rates of convergence (1.4)-(1.7) in Theorem 1.1 is based on Proposition 2.2 with the help of the bootstrap argument that will be shown in Subsection 4.3.

2.2. A priori estimates. In this subsection we devote ourselves to the proof of Proposition 2.1. The key part is to apply the classical energy method to obtain some uniform-in-time $a$ priori estimates for smooth solutions to the Cauchy problem (2.2)-(2.3). Notice that (2.2) is a quasi-linear symmetric hyperbolic system.

Theorem 2.1. (a priori estimates). Let $0<T \leq \infty$ be given. Suppose that $V=\left[\sigma_{ \pm}, v_{ \pm}, \tilde{E}, \tilde{B}\right] \in$ $C\left([0, T) ; H^{N}\left(\mathbb{R}^{3}\right)\right)$ is smooth with

$$
\sup _{0 \leq t<T}\left\|\sigma_{ \pm}(t)\right\|_{N} \leq 1
$$


and that $V$ satisfies the system (2.2) for $t \in(0, T)$. Then, there are $\mathcal{E}_{N}(\cdot)$ and $\mathcal{D}_{N}(\cdot)$ in the form (2.5) and (2.7) such that

$$
\frac{d}{d t} \mathcal{E}_{N}(V(t))+\lambda \mathcal{D}_{N}(V(t)) \leq C\left[\mathcal{E}_{N}(V(t))^{\frac{1}{2}}+\mathcal{E}_{N}(V(t))\right] \mathcal{D}_{N}(V(t))
$$

for any $0 \leq t<T$.

Proof. It is divided by five steps as follows.

Step 1. It holds that

$$
\frac{1}{2} \frac{d}{d t}\|V\|_{N}^{2}+\frac{1}{\sqrt{\gamma}}\left\|\left[v_{+}, v_{-}\right]\right\|_{N}^{2} \leq C\|V\|_{N}\left(\left\|\left[v_{+}, v_{-}\right]\right\|^{2}+\left\|\nabla\left[\sigma_{+}, \sigma_{-}, v_{+}, v_{-}\right]\right\|_{N-1}^{2}\right) .
$$

In fact, from the first two equations of (2.2), energy estimates on $\partial^{\alpha} \sigma_{ \pm}$and $\partial^{\alpha} v_{ \pm}$with $|\alpha| \leq N$ give

$$
\begin{aligned}
& \frac{1}{2} \frac{d}{d t}\left\|\partial^{\alpha}\left[\sigma_{+}, \sigma_{-}, v_{+}, v_{-}\right]\right\|^{2}+\frac{1}{\sqrt{\gamma}}\left\|\partial^{\alpha}\left[v_{+}, v_{-}\right]\right\|^{2}+\frac{1}{\sqrt{\gamma}}\left\langle\partial^{\alpha} \tilde{E}, \partial^{\alpha} v_{+}\right\rangle \\
& -\frac{1}{\sqrt{\gamma}}\left\langle\partial^{\alpha} \tilde{E}, \partial^{\alpha} v_{-}\right\rangle=-\sum_{\beta<\alpha} C_{\beta}^{\alpha} I_{\alpha, \beta}(t)+I_{1}(t) .
\end{aligned}
$$

Here, $I_{\alpha, \beta}(t)=I_{\alpha, \beta}^{+}(t)+I_{\alpha, \beta}^{-}(t), I_{1}(t)=I_{1}^{+}(t)+I_{1}^{-}(t)$ with

$$
\begin{aligned}
I_{\alpha, \beta}^{+}(t)= & \left\langle\partial^{\alpha-\beta} v_{+} \cdot \nabla \partial^{\beta} \sigma_{+}, \partial^{\alpha} \sigma_{+}\right\rangle+\frac{\gamma-1}{2}\left\langle\partial^{\alpha-\beta} \sigma_{+} \nabla \cdot \partial^{\beta} v_{+}, \partial^{\alpha} \sigma_{+}\right\rangle \\
& +\frac{\gamma-1}{2}\left\langle\partial^{\alpha-\beta} \sigma_{+} \nabla \partial^{\beta} \sigma_{+}, \partial^{\alpha} v_{+}\right\rangle+\left\langle\partial^{\alpha-\beta} v_{+} \cdot \nabla \partial^{\beta} v_{+}, \partial^{\alpha} v_{+}\right\rangle \\
& +\left\langle\partial^{\alpha-\beta} v_{+} \times \partial^{\beta} \tilde{B}, \partial^{\alpha} v_{+}\right\rangle, \\
I_{\alpha, \beta}^{-}(t)= & \left\langle\partial^{\alpha-\beta} v_{-} \cdot \nabla \partial^{\beta} \sigma_{-}, \partial^{\alpha} \sigma_{-}\right\rangle+\frac{\gamma-1}{2}\left\langle\partial^{\alpha-\beta} \sigma_{-} \nabla \cdot \partial^{\beta} v_{-}, \partial^{\alpha} \sigma_{-}\right\rangle \\
& +\frac{\gamma-1}{2}\left\langle\partial^{\alpha-\beta} \sigma_{-} \nabla \partial^{\beta} \sigma_{-}, \partial^{\alpha} v_{-}\right\rangle+\left\langle\partial^{\alpha-\beta} v_{-} \cdot \nabla \partial^{\beta} v_{-}, \partial^{\alpha} v_{-}\right\rangle \\
& -\left\langle\partial^{\alpha-\beta} v_{-} \times \partial^{\beta} \tilde{B}, \partial^{\alpha} v_{-}\right\rangle,
\end{aligned}
$$

and

$$
\begin{aligned}
& I_{1}^{+}(t)=\frac{1}{2}\left\langle\nabla \cdot v_{+},\left|\partial^{\alpha} \sigma_{+}\right|^{2}+\left|\partial^{\alpha} v_{+}\right|^{2}\right\rangle+\frac{\gamma-1}{2}\left\langle\nabla \sigma_{+} \cdot \partial^{\alpha} \sigma_{+}, \partial^{\alpha} v_{+}\right\rangle-\left\langle v_{+} \times \partial^{\alpha} \tilde{B}, \partial^{\alpha} v_{+}\right\rangle, \\
& I_{1}^{-}(t)=\frac{1}{2}\left\langle\nabla \cdot v_{-},\left|\partial^{\alpha} \sigma_{-}\right|^{2}+\left|\partial^{\alpha} v_{-}\right|^{2}\right\rangle+\frac{\gamma-1}{2}\left\langle\nabla \sigma_{-} \cdot \partial^{\alpha} \sigma_{-}, \partial^{\alpha} v_{-}\right\rangle+\left\langle v_{-} \times \partial^{\alpha} \tilde{B}, \partial^{\alpha} v_{-}\right\rangle,
\end{aligned}
$$

where integration by parts was used. When $|\alpha|=0$, it suffices to estimate $I_{1}(t)$ by

$$
\begin{aligned}
I_{1}(t)= & I_{1}^{+}(t)+I_{1}^{-}(t) \\
\leq & C\left\|\left[\sigma_{+}, v_{+}\right]\right\|_{H^{1}}\left\|\nabla\left[\sigma_{+}, v_{+}\right]\right\|^{2}+C\|\nabla \tilde{B}\|_{H^{1}}\left\|v_{+}\right\|^{2} \\
& +C\left\|\left[\sigma_{-}, v_{-}\right]\right\|_{H^{1}}\left\|\nabla\left[\sigma_{-}, v_{-}\right]\right\|^{2}+C\|\nabla \tilde{B}\|_{H^{1}}\left\|v_{-}\right\|^{2} \\
\leq & C\left\|\left[\sigma_{+}, \sigma_{-}, v_{+}, v_{-}\right]\right\|_{H^{1}}\left\|\nabla\left[\sigma_{+}, \sigma_{-}, v_{+}, v_{-}\right]\right\|^{2}+C\|\nabla \tilde{B}\|_{H^{1}}\left\|\left[v_{+}, v_{-}\right]\right\|^{2},
\end{aligned}
$$

which is further bounded by the r.h.s. term of (2.16). When $|\alpha| \geq 1$, since each term in $I_{\alpha, \beta}(t)$ and $I_{1}(t)$ is the integration of the three-terms product in which there is at least one term containing the derivative, one has

$$
I_{\alpha, \beta}(t)+I_{1}(t) \leq C\left\|\left[\sigma_{+}, \sigma_{-}, v_{+}, v_{-}, \tilde{B}\right]\right\|_{N}\left\|\nabla\left[\sigma_{+}, \sigma_{-}, v_{+}, v_{-}\right]\right\|_{N-1}^{2},
$$


which is also bounded by the r.h.s. term of (2.16). On the other hand, from (2.2), energy estimates on $\partial^{\alpha} \tilde{E}$ and $\partial^{\alpha} \tilde{B}$ with $|\alpha| \leq N$ give

$$
\begin{aligned}
& \frac{1}{2} \frac{d}{d t}\left\|\partial^{\alpha}[\tilde{E}, \tilde{B}]\right\|^{2}-\frac{1}{\sqrt{\gamma}}\left\langle\partial^{\alpha} v_{+}, \partial^{\alpha} \tilde{E}\right\rangle+\frac{1}{\sqrt{\gamma}}\left\langle\partial^{\alpha} \tilde{E}, \partial^{\alpha} v_{-}\right\rangle \\
= & \frac{1}{\sqrt{\gamma}}\left\langle\partial^{\alpha}\left[\left(\Phi\left(\sigma_{+}\right)+\sigma_{+}\right) v_{+}\right], \partial^{\alpha} \tilde{E}\right\rangle-\frac{1}{\sqrt{\gamma}}\left\langle\partial^{\alpha}\left[\left(\Phi\left(\sigma_{-}\right)+\sigma_{-}\right) v_{-}\right], \partial^{\alpha} \tilde{E}\right\rangle \\
:= & I_{2}^{+}(t)+I_{2}^{-}(t) .
\end{aligned}
$$

In a similar way as [6], for $|\alpha| \leq N$, one has

$$
I_{2}^{+}(t) \leq C\|\tilde{E}\|_{N}\left(\left\|\nabla\left[\sigma_{+}, v_{+}\right]\right\|_{N-1}^{2}+\left\|v_{+}\right\|^{2}\right),
$$

and

$$
I_{2}^{-}(t) \leq C\|\tilde{E}\|_{N}\left(\left\|\nabla\left[\sigma_{-}, v_{-}\right]\right\|_{N-1}^{2}+\left\|v_{-}\right\|^{2}\right) .
$$

Thus, for $|\alpha| \leq N$, one has

$$
I_{2}^{+}(t)+I_{2}^{-}(t) \leq C\|\tilde{E}\|_{N}\left(\left\|\nabla\left[\sigma_{+}, \sigma_{-}, v_{+}, v_{-}\right]\right\|_{N-1}^{2}+\left\|\left[v_{+}, v_{-}\right]\right\|^{2}\right),
$$

which is bounded by the r.h.s. term of (2.16). Then (2.16) follows by taking summation of (2.17) and (2.18) over $|\alpha| \leq N$.

Step 2. It holds that

$$
\begin{aligned}
& \frac{d}{d t} \mathcal{E}_{N, 1}^{i n t}(V)+\lambda\left(\left\|\nabla\left[\sigma_{+}, \sigma_{-}\right]\right\|_{N-1}^{2}+\left\|\sigma_{+}-\sigma_{-}\right\|^{2}\right) \\
\leq & C\left\|\left[v_{+}, v_{-}\right]\right\|_{N}^{2}+C\left\|\left[\sigma_{+}, \sigma_{-}, v_{+}, v_{-}, \tilde{B}\right]\right\|_{N}^{2}\left\|\nabla\left[\sigma_{+}, \sigma_{-}, v_{+}, v_{-}\right]\right\|_{N-1}^{2},
\end{aligned}
$$

where $\mathcal{E}_{N, 1}^{\text {int }}(\cdot)$ is defined by

$$
\mathcal{E}_{N, 1}^{i n t}(V)=\sum_{|\alpha| \leq N-1}\left(\left\langle\partial^{\alpha} v_{+}, \nabla \partial^{\alpha} \sigma_{+}\right\rangle+\left\langle\partial^{\alpha} v_{-}, \nabla \partial^{\alpha} \sigma_{-}\right\rangle\right) .
$$

In fact, the first four equations of (2.2) can be rewritten as

$$
\begin{gathered}
\partial_{t} \sigma_{+}+\nabla \cdot v_{+}=f_{1}^{+}, \\
\partial_{t} \sigma_{-}+\nabla \cdot v_{-}=f_{1}^{-}, \\
\partial_{t} v_{+}+\nabla \sigma_{+}+\frac{1}{\sqrt{\gamma}} \tilde{E}=f_{2}^{+}-\frac{1}{\sqrt{\gamma}} v_{+}, \\
\partial_{t} v_{-}+\nabla \sigma_{-}-\frac{1}{\sqrt{\gamma}} \tilde{E}=f_{2}^{-}-\frac{1}{\sqrt{\gamma}} v_{-},
\end{gathered}
$$

where

$$
\left\{\begin{array}{l}
f_{1}^{+}=-v_{+} \cdot \nabla \sigma_{+}-\frac{\gamma-1}{2} \sigma_{+} \nabla \cdot v_{+}, \\
f_{1}^{-}=-v_{-} \cdot \nabla \sigma_{-}-\frac{\gamma-1}{2} \sigma_{-} \nabla \cdot v_{-}, \\
f_{2}^{+}=-v_{+} \cdot \nabla v_{+}-\frac{\gamma-1}{2} \sigma_{+} \nabla \sigma_{+}-v_{+} \times \tilde{B}, \\
f_{2}^{-}=-v_{-} \cdot \nabla v_{-}-\frac{\gamma-1}{2} \sigma_{-} \nabla \sigma_{-}+v_{-} \times \tilde{B} .
\end{array}\right.
$$


Let $|\alpha| \leq N-1$. Applying $\partial^{\alpha}$ to (2.23), multiplying it by $\partial^{\alpha} \nabla \sigma_{-}$, taking integrations in $x$ and then using integration by parts and also the final equation of (2.2), replacing $\partial_{t} \sigma_{-}$from (2.21) gives

$$
\begin{aligned}
& \frac{d}{d t}\left\langle\partial^{\alpha} v_{-}, \nabla \partial^{\alpha} \sigma_{-}\right\rangle+\left\|\nabla \partial^{\alpha} \sigma_{-}\right\|^{2}+\frac{1}{\gamma}\left\|\partial^{\alpha} \sigma_{-}\right\|^{2}-\frac{1}{\gamma}\left\langle\partial^{\alpha} \sigma_{+}, \partial^{\alpha} \sigma_{-}\right\rangle \\
= & -\frac{1}{\gamma}\left\langle\partial^{\alpha} \Phi\left(\sigma_{-}\right), \partial^{\alpha} \sigma_{-}\right\rangle+\frac{1}{\gamma}\left\langle\partial^{\alpha} \Phi\left(\sigma_{+}\right), \partial^{\alpha} \sigma_{-}\right\rangle+\left\langle\partial^{\alpha} f_{2}^{-}, \nabla \partial^{\alpha} \sigma_{-}\right\rangle \\
& -\frac{1}{\sqrt{\gamma}}\left\langle\partial^{\alpha} v_{-}, \nabla \partial^{\alpha} \sigma_{-}\right\rangle+\left\|\nabla \cdot \partial^{\alpha} v_{-}\right\|^{2}-\left\langle\partial^{\alpha} f_{1}^{-}, \nabla \cdot \partial^{\alpha} v_{-}\right\rangle .
\end{aligned}
$$

In a similar way as above, from (2.22) and (2.20), one has

$$
\begin{aligned}
& \frac{d}{d t}\left\langle\partial^{\alpha} v_{+}, \nabla \partial^{\alpha} \sigma_{+}\right\rangle+\left\|\nabla \partial^{\alpha} \sigma_{+}\right\|^{2}+\frac{1}{\gamma}\left\|\partial^{\alpha} \sigma_{+}\right\|^{2}-\frac{1}{\gamma}\left\langle\partial^{\alpha} \sigma_{-}, \partial^{\alpha} \sigma_{+}\right\rangle \\
= & -\frac{1}{\gamma}\left\langle\partial^{\alpha} \Phi\left(\sigma_{+}\right), \partial^{\alpha} \sigma_{+}\right\rangle+\frac{1}{\gamma}\left\langle\partial^{\alpha} \Phi\left(\sigma_{-}\right), \partial^{\alpha} \sigma_{+}\right\rangle+\left\langle\partial^{\alpha} f_{2}^{+}, \nabla \partial^{\alpha} \sigma_{+}\right\rangle \\
& -\frac{1}{\sqrt{\gamma}}\left\langle\partial^{\alpha} v_{+}, \nabla \partial^{\alpha} \sigma_{+}\right\rangle+\left\|\nabla \cdot \partial^{\alpha} v_{+}\right\|^{2}-\left\langle\partial^{\alpha} f_{1}^{+}, \nabla \cdot \partial^{\alpha} v_{+}\right\rangle .
\end{aligned}
$$

Taking further the summation of the previous two equations implies

$$
\begin{aligned}
& \frac{d}{d t}\left(\left\langle\partial^{\alpha} v_{+}, \nabla \partial^{\alpha} \sigma_{+}\right\rangle+\left\langle\partial^{\alpha} v_{-}, \nabla \partial^{\alpha} \sigma_{-}\right\rangle\right)+\left\|\nabla \partial^{\alpha}\left[\sigma_{+}, \sigma_{-}\right]\right\|^{2}+\frac{1}{\gamma}\left\|\partial^{\alpha} \sigma_{+}-\partial^{\alpha} \sigma_{-}\right\|^{2} \\
= & \left\|\nabla \cdot \partial^{\alpha} v_{+}\right\|^{2}+\left\|\nabla \cdot \partial^{\alpha} v_{-}\right\|^{2}+\frac{1}{\gamma}\left\langle\partial^{\alpha} \Phi\left(\sigma_{+}\right), \partial^{\alpha} \sigma_{-}-\partial^{\alpha} \sigma_{+}\right\rangle+\left\langle\partial^{\alpha} f_{2}^{+}, \nabla \partial^{\alpha} \sigma_{+}\right\rangle \\
& +\left\langle\partial^{\alpha} f_{2}^{-}, \nabla \partial^{\alpha} \sigma_{-}\right\rangle+\frac{1}{\gamma}\left\langle\partial^{\alpha} \Phi\left(\sigma_{-}\right), \partial^{\alpha} \sigma_{+}-\partial^{\alpha} \sigma_{-}\right\rangle-\frac{1}{\sqrt{\gamma}}\left\langle\partial^{\alpha} v_{+}, \nabla \partial^{\alpha} \sigma_{+}\right\rangle \\
& -\frac{1}{\sqrt{\gamma}}\left\langle\partial^{\alpha} v_{-}, \nabla \partial^{\alpha} \sigma_{-}\right\rangle-\left\langle\partial^{\alpha} f_{1}^{+}, \nabla \cdot \partial^{\alpha} v_{+}\right\rangle-\left\langle\partial^{\alpha} f_{1}^{-}, \nabla \cdot \partial^{\alpha} v_{-}\right\rangle .
\end{aligned}
$$

Then, it follows from Cauchy-Schwarz inequality that

$$
\begin{aligned}
& \frac{d}{d t}\left(\left\langle\partial^{\alpha} v_{+}, \nabla \partial^{\alpha} \sigma_{+}\right\rangle+\left\langle\partial^{\alpha} v_{-}, \nabla \partial^{\alpha} \sigma_{-}\right\rangle\right)+\lambda\left(\left\|\nabla \partial^{\alpha}\left[\sigma_{+}, \sigma_{-}\right]\right\|^{2}+\left\|\partial^{\alpha} \sigma_{+}-\partial^{\alpha} \sigma_{-}\right\|^{2}\right) \\
\leq & C\left(\left\|\nabla \cdot \partial^{\alpha} v_{+}\right\|^{2}+\left\|\nabla \cdot \partial^{\alpha} v_{-}\right\|^{2}\right)+C\left(\left\|\partial^{\alpha} v_{+}\right\|^{2}+\left\|\partial^{\alpha} v_{-}\right\|^{2}\right) \\
& +C\left(\left\|\partial^{\alpha} \Phi\left(\sigma_{+}\right)\right\|^{2}+\left\|\partial^{\alpha} f_{1}^{+}\right\|^{2}+\left\|\partial^{\alpha} f_{2}^{+}\right\|^{2}\right)+C\left(\left\|\partial^{\alpha} \Phi\left(\sigma_{-}\right)\right\|^{2}+\left\|\partial^{\alpha} f_{1}^{-}\right\|^{2}+\left\|\partial^{\alpha} f_{2}^{-}\right\|^{2}\right) .
\end{aligned}
$$

Noticing that $\Phi(\sigma)$ is smooth in $\sigma$ with $\Phi(0)=\Phi^{\prime}(0)=0$ and $f_{1}^{+}, f_{1}^{-}, f_{2}^{+}, f_{2}^{-}$are quadratically nonlinear, one has from (2.14) that

$$
\begin{aligned}
& \left\|\partial^{\alpha} \Phi\left(\sigma_{+}\right)\right\|^{2}+\left\|\partial^{\alpha} f_{1}^{+}\right\|^{2}+\left\|\partial^{\alpha} f_{2}^{+}\right\|^{2}+\left\|\partial^{\alpha} \Phi\left(\sigma_{-}\right)\right\|^{2}+\left\|\partial^{\alpha} f_{1}^{-}\right\|^{2}+\left\|\partial^{\alpha} f_{2}^{-}\right\|^{2} \\
\leq & C\left\|\left[\sigma_{+}, v_{+}, \tilde{B}\right]\right\|_{N}^{2}\left\|\nabla\left[\sigma_{+}, v_{+}\right]\right\|_{N-1}^{2}+C\left\|\left[\sigma_{-}, v_{-}, \tilde{B}\right]\right\|_{N}^{2}\left\|\nabla\left[\sigma_{-}, v_{-}\right]\right\|_{N-1}^{2} .
\end{aligned}
$$

Plugging this into (2.24) taking summation over $|\alpha| \leq N-1$ yields (2.19).

Step 3. It holds that

$$
\begin{aligned}
& \frac{d}{d t} \mathcal{E}_{N, 2}^{i n t}(V)+\lambda\|\tilde{E}\|_{N-1}^{2} \\
\leq & C\left\|\left[v_{+}, v_{-}\right]\right\|_{N}^{2}+C\left\|\nabla\left[\sigma_{+}, \sigma_{-}\right]\right\|_{N-1}^{2}+C\left\|\left[v_{+}, v_{-}\right]\right\|_{N}\|\nabla \tilde{B}\|_{N-2} \\
& +C\left\|\left[\sigma_{+}, \sigma_{-}, v_{+}, v_{-}, \tilde{B}\right]\right\|_{N}^{2}\left\|\nabla\left[\sigma_{+}, \sigma_{-}, v_{+}, v_{-}\right]\right\|_{N-1}^{2},
\end{aligned}
$$


where $\mathcal{E}_{N, 2}^{\text {int }}(\cdot)$ is defined by

$$
\mathcal{E}_{N, 2}^{i n t}(V)=\sum_{|\alpha| \leq N-1}\left\langle\partial^{\alpha}\left(v_{+}-v_{-}\right), \partial^{\alpha} \tilde{E}\right\rangle .
$$

In fact, for $|\alpha| \leq N-1$, from (2.22) and (2.23), we have

$$
\begin{aligned}
& \partial_{t}\left(v_{+}-v_{-}\right)+\left(\nabla \sigma_{+}-\nabla \sigma_{-}\right)+\frac{2}{\sqrt{\gamma}} \tilde{E} \\
= & f_{2}^{+}-f_{2}^{-}-\frac{1}{\sqrt{\gamma}}\left(v_{+}-v_{-}\right) .
\end{aligned}
$$

Applying $\partial^{\alpha}$ to $(2.26)$, multiplying it by $\partial^{\alpha} \tilde{E}$, taking integrations in $x$ and using integration by parts and replacing $\partial_{t} \tilde{E}$ from the third equation of (2.2) gives

$$
\begin{aligned}
& \frac{d}{d t}\left\langle\partial^{\alpha}\left(v_{+}-v_{-}\right), \partial^{\alpha} \tilde{E}\right\rangle+\frac{2}{\sqrt{\gamma}}\left\|\partial^{\alpha} \tilde{E}\right\|^{2} \\
= & \frac{1}{\sqrt{\gamma}}\left\|\partial^{\alpha}\left(v_{+}-v_{-}\right)\right\|^{2}+\frac{1}{\sqrt{\gamma}}\left\langle\partial^{\alpha}\left(v_{+}-v_{-}\right), \nabla \times \partial^{\alpha} \tilde{B}\right\rangle \\
& +\frac{1}{\sqrt{\gamma}}\left\langle\partial^{\alpha}\left(v_{+}-v_{-}\right), \partial^{\alpha}\left[\Phi\left(\sigma_{+}\right) v_{+}+\sigma_{+} v_{+}\right]\right\rangle \\
& -\frac{1}{\sqrt{\gamma}}\left\langle\partial^{\alpha}\left(v_{+}-v_{-}\right), \partial^{\alpha}\left[\Phi\left(\sigma_{-}\right) v_{-}+\sigma_{-} v_{-}\right]\right\rangle-\frac{1}{\sqrt{\gamma}}\left\langle\partial^{\alpha}\left(v_{+}-v_{-}\right), \partial^{\alpha} \tilde{E}\right\rangle \\
& -\left\langle\partial^{\alpha}\left(\nabla \sigma_{+}-\nabla \sigma_{-}\right), \partial^{\alpha} \tilde{E}\right\rangle+\left\langle\partial^{\alpha}\left(f_{2}^{+}-f_{2}^{-}\right), \partial^{\alpha} \tilde{E}\right\rangle,
\end{aligned}
$$

which from Cauchy-Schwarz inequality, further implies

$$
\begin{aligned}
& \frac{d}{d t}\left\langle\partial^{\alpha}\left(v_{+}-v_{-}\right), \partial^{\alpha} \tilde{E}\right\rangle+\lambda\left\|\partial^{\alpha} \tilde{E}\right\|^{2} \\
\leq & C\left\|\partial^{\alpha}\left(v_{+}-v_{-}\right)\right\|^{2}+C\left\|\left[v_{+}, v_{-}\right]\right\|_{N}\|\nabla \tilde{B}\|_{N-2}+C\left\|\nabla\left[\partial^{\alpha} \sigma_{+}-\partial^{\alpha} \sigma_{-}\right]\right\|^{2} \\
& +C\left\|\left[\sigma_{+}, \sigma_{-}, v_{+}, v_{-}, \tilde{B}\right]\right\|_{N}^{2}\left\|\nabla\left[\sigma_{+}, \sigma_{-}, v_{+}, v_{-}\right]\right\|_{N-1}^{2} .
\end{aligned}
$$

Thus (2.25) follows from taking summation of the above estimate over $|\alpha| \leq N-1$.

Step 4. It holds that

$$
\begin{aligned}
\frac{d}{d t} \mathcal{E}_{N, 3}^{i n t}(V)+\lambda\|\nabla \tilde{B}\|_{N-2}^{2} \leq & C\left\|\left[v_{+}, v_{-}, \tilde{E}\right]\right\|_{N-1}^{2} \\
& +C\left\|\left[\sigma_{+}, \sigma_{-}\right]\right\|_{N}^{2}\left\|\nabla\left[v_{+}, v_{-}\right]\right\|_{N-1}^{2},
\end{aligned}
$$

where $\mathcal{E}_{N, 3}^{\text {int }}(\cdot)$ is defined by

$$
\mathcal{E}_{N, 3}^{i n t}(V)=-\sum_{|\alpha| \leq N-2}\left\langle\nabla \times \partial^{\alpha} \tilde{E}, \partial^{\alpha} \tilde{B}\right\rangle
$$

In fact, for $|\alpha| \leq N-2$, applying $\partial^{\alpha}$ to the third equation of (2.2), multiplying it by $\partial^{\alpha} \nabla \times \tilde{B}$, taking integrations in $x$ and using integration by parts and replacing $\partial_{t} \tilde{B}$ from the fourth equation of (2.2) implies

$$
\begin{aligned}
& -\frac{d}{d t}\left\langle\partial^{\alpha} \tilde{E}, \nabla \times \partial^{\alpha} \tilde{B}\right\rangle+\frac{1}{\sqrt{\gamma}}\left\|\nabla \times \partial^{\alpha} \tilde{B}\right\|^{2} \\
= & \frac{1}{\sqrt{\gamma}}\left\|\nabla \times \partial^{\alpha} \tilde{E}\right\|^{2}-\frac{1}{\sqrt{\gamma}}\left\langle\partial^{\alpha} v_{+}, \nabla \times \partial^{\alpha} \tilde{B}\right\rangle+\frac{1}{\sqrt{\gamma}}\left\langle\partial^{\alpha} v_{-}, \nabla \times \partial^{\alpha} \tilde{B}\right\rangle \\
& -\frac{1}{\sqrt{\gamma}}\left\langle\partial^{\alpha}\left[\Phi\left(\sigma_{+}\right) v_{+}+\sigma_{+} v_{+}\right], \nabla \times \partial^{\alpha} \tilde{B}\right\rangle+\frac{1}{\sqrt{\gamma}}\left\langle\partial^{\alpha}\left[\Phi\left(\sigma_{-}\right) v_{-}+\sigma_{-} v_{-}\right], \nabla \times \partial^{\alpha} \tilde{B}\right\rangle .
\end{aligned}
$$


The above estimate gives (2.27) by further using Cauchy-Schwarz inequality and taking summation over $|\alpha| \leq N-2$, where we also used

$$
\left\|\partial^{\alpha} \partial_{x_{i}} \tilde{B}\right\|=\left\|\partial_{x_{i}} \Delta^{-1} \nabla \times\left(\nabla \times \partial^{\alpha} \tilde{B}\right)\right\| \leq\left\|\nabla \times \partial^{\alpha} \tilde{B}\right\|
$$

for each $1 \leq i \leq 3$, due to the fact that $\partial_{x_{i}} \Delta^{-1} \nabla$ is bounded from $L^{p}$ to itself for $1<p<\infty$ [19].

Step 5. Now, following four steps above, we are ready to prove (2.15). Let us define

$$
\mathcal{E}_{N}(V(t))=\|V(t)\|_{N}^{2}+\sum_{i=1}^{3} \kappa_{i} \mathcal{E}_{N, i}^{i n t}(V(t)),
$$

that is,

$$
\begin{aligned}
\mathcal{E}_{N}(V(t))= & \left\|\left[\sigma_{+}, \sigma_{-}, v_{+}, v_{-}, \tilde{E}, \tilde{B}\right]\right\|_{N}^{2} \\
& +\kappa_{1} \sum_{|\alpha| \leq N-1}\left(\left\langle\partial^{\alpha} v_{+}, \nabla \partial^{\alpha} \sigma_{+}\right\rangle+\left\langle\partial^{\alpha} v_{-}, \nabla \partial^{\alpha} \sigma_{-}\right\rangle\right) \\
& +\kappa_{2} \sum_{|\alpha| \leq N-1}\left\langle\partial^{\alpha}\left(v_{+}-v_{-}\right), \nabla \partial^{\alpha} \tilde{E}\right\rangle-\kappa_{3} \sum_{|\alpha| \leq N-2}\left\langle\nabla \times \partial^{\alpha} \tilde{E}, \partial^{\alpha} \tilde{B}\right\rangle
\end{aligned}
$$

for constants $0<\kappa_{3} \ll \kappa_{2} \ll \kappa_{1} \ll 1$ to be determined. Notice that as long as $0<\kappa_{i} \ll 1$ is small enough for $i=1,2,3$, then $\mathcal{E}_{N}(V(t)) \sim\|V(t)\|_{N}^{2}$ holds true. Moreover, letting $0<\kappa_{3} \ll \kappa_{2} \ll \kappa_{1} \ll 1$ with $\kappa_{2}^{3 / 2} \ll \kappa_{3}$, the sum of $(2.16),(2.19) \times \kappa_{1},(2.25) \times \kappa_{2},(2.27) \times \kappa_{3}$ implies that there is $\lambda>0, C>0$ such that $(2.15)$ holds true with $\mathcal{D}_{N}(\cdot)$ defined in (2.7). Here, we have used the following Cauchy-Schwarz inequality

$$
2 \kappa_{2}\left\|\left[v_{+}, v_{-}\right]\right\|_{N}\|\nabla \tilde{B}\|_{N-2} \leq \kappa_{2}^{1 / 2}\left\|\left[v_{+}, v_{-}\right]\right\|_{N}^{2}+\kappa_{2}^{3 / 2}\|\nabla \tilde{B}\|_{N-2}^{2} .
$$

and due to $\kappa_{2}^{3 / 2} \ll \kappa_{3}$, both terms on the r.h.s. of the above inequality were absorbed. This completes the proof of Theorem 2.1.

Since (2.2) is a quasi-linear symmetric hyperbolic system, the short-time existence can be proved in much more general case as in [13]; see also ([20] Theorem 1.2, Proposition 1.3 and Proposition 1.4 in Chapter 16). From Theorem 2.1 and the continuity argument, it is easy to see that $\mathcal{E}_{N}(V(t))$ is bounded uniformly in time under the assumption that $\mathcal{E}_{N}\left(V_{0}\right)>0$ is small enough. Therefore, the global existence of solutions satisfying (2.9) and (2.10) follows in the standard way; see also [6]. This completes the proof of Proposition 2.1.

\section{LINEARIZED HOMOGENEOUS SYSTEM}

In order to study the time-decay property of solutions to the nonlinear system (1.1) later on, we have to consider the following Cauchy problem on the corresponding linearized system around the constant state $[1,0,0,0]$. In fact, by setting $\rho_{ \pm}=n_{ \pm}-1$, Then $U:=\left[\rho_{ \pm}, u_{ \pm}, E, B\right]$ satisfies

$$
\left\{\begin{array}{l}
\partial_{t} \rho_{ \pm}+\nabla \cdot u_{ \pm}=-\nabla \cdot\left(\rho_{ \pm} u_{ \pm}\right), \\
\partial_{t} u_{ \pm}+u_{ \pm} \pm E+\gamma \nabla \rho_{ \pm}=-u_{ \pm} \cdot \nabla u_{ \pm}-\gamma\left[\left(\rho_{ \pm}+1\right)^{\gamma-2}-1\right] \nabla \rho_{ \pm} \mp\left(u_{ \pm} \times B\right), \\
\partial_{t} E-\nabla \times B-u_{+}+u_{-}=\rho_{+} u_{+}-\rho_{-} u_{-}, \\
\partial_{t} B+\nabla \times E=0, \\
\nabla \cdot E=\rho_{-}-\rho_{+}, \quad \nabla \cdot B=0, \quad t>0, x \in \mathbb{R}^{3},
\end{array}\right.
$$

with initial data

$$
\left.U\right|_{t=0}=U_{0}:=\left[\rho_{ \pm 0}, u_{ \pm 0}, E_{0}, B_{0}\right], \quad x \in \mathbb{R}^{3},
$$


satisfying the compatibility conditions $\nabla \cdot E_{0}=\rho_{-0}-\rho_{+0}, \nabla \cdot B_{0}=0$. Here, $\rho_{ \pm 0}=n_{ \pm 0}-1$.

To capture the decay structure of the linearized system above, we now take the sum and difference of \pm equations. Let

$$
\rho_{1}=\frac{\rho_{+}-\rho_{-}}{2}, \quad u_{1}=\frac{u_{+}-u_{-}}{2} .
$$

Then $\left[\rho_{1}, u_{1}, E, B\right]$ satisfies

$$
\left\{\begin{array}{l}
\partial_{t} \rho_{1}+\nabla \cdot u_{1}=\frac{1}{2}\left(g_{1}^{+}-g_{1}^{-}\right), \\
\partial_{t} u_{1}+u_{1}+E+\gamma \nabla \rho_{1}=\frac{1}{2}\left(g_{2}^{+}-g_{2}^{-}\right), \\
\partial_{t} E-\nabla \times B-2 u_{1}=\frac{1}{2}\left(g_{3}^{+}-g_{3}^{-}\right), \\
\partial_{t} B+\nabla \times E=0, \\
\frac{1}{2} \nabla \cdot E=-\rho_{1}, \quad \nabla \cdot B=0, \quad t>0, x \in \mathbb{R}^{3},
\end{array}\right.
$$

with initial data $\left.U_{1}\right|_{t=0}=U_{1,0}:=\left[\rho_{1,0}, u_{1,0}, E_{0}, B_{0}\right], x \in \mathbb{R}^{3}$, satisfying the compatibility conditions $\frac{1}{2} \nabla \cdot E=-\rho_{1,0}, \nabla \cdot B_{0}=0$, and $\left[\rho_{1,0}, u_{1,0}\right]$ is given from $\left[\rho_{+0}, \rho_{-0}, u_{+0}, u_{-0}\right]$ according to the transform (3.3). Furthermore, we also set

$$
\rho_{2}=\frac{\rho_{+}+\rho_{-}}{2}, \quad u_{2}=\frac{u_{+}+u_{-}}{2} .
$$

Then $\left[\rho_{2}, u_{2}\right]$ satisfies

$$
\left\{\begin{array}{l}
\partial_{t} \rho_{2}+\nabla \cdot u_{2}=\frac{1}{2}\left(g_{1}^{+}+g_{1}^{-}\right), \\
\partial_{t} u_{2}+u_{2}+\gamma \nabla \rho_{2}=\frac{1}{2}\left(g_{2}^{+}+g_{2}^{-}\right), \quad t>0, x \in \mathbb{R}^{3},
\end{array}\right.
$$

with initial data $\left.U_{2}\right|_{t=0}=U_{2,0}:=\left[\rho_{2,0}, u_{2,0}\right], x \in \mathbb{R}^{3}$, where $\left[\rho_{2,0}, u_{2,0}\right]$ is given from $\left[\rho_{+0}, \rho_{-0}, u_{+0}, u_{0}\right]$ according to the transform (3.5). Here the nonlinear source term takes the form of

$$
\left\{\begin{array}{l}
g_{1}^{ \pm}=-\nabla \cdot\left(\rho_{ \pm} u_{ \pm}\right), \\
g_{2}^{ \pm}=-u_{ \pm} \cdot \nabla u_{ \pm} \mp u_{ \pm} \times B-\gamma\left[\left(1+\rho_{ \pm}\right)^{\gamma-2}-1\right] \nabla \rho_{ \pm}, \\
g_{3}^{ \pm}=\rho_{ \pm} u_{ \pm}
\end{array}\right.
$$

Then, by Duhamel's principle, the solution $U_{1}=\left[\rho_{1}, u_{1}, E, B\right]$ and $U_{2}=\left[\rho_{2}, u_{2}\right]$ can be formally written as

$$
U_{1}(t)=e^{t L} U_{1,0}+\frac{1}{2} \int_{0}^{t} e^{(t-s) L}\left[g_{1}^{+}(s)-g_{1}^{-}(s), g_{2}^{+}(s)-g_{2}^{-}(s), g_{3}^{+}(s)-g_{3}^{-}(s), 0\right] d s
$$

and

$$
U_{2}(t)=e^{t L} U_{2,0}+\frac{1}{2} \int_{0}^{t} e^{(t-s) L}\left[g_{1}^{+}(s)+g_{1}^{-}(s), g_{2}^{+}(s)+g_{2}^{-}(s)\right] d s,
$$

where $e^{t L} U_{1,0}$ and $e^{t L} U_{2,0}$, respectively, denote the solution to the Cauchy problems (3.10)(3.11) and (3.13)-(3.14) both without nonlinear sources, which will be given later on.

Thus, in this section, we are concerned with the time-decay estimates on two decoupled linearized homogeneous systems corresponding to (3.4) and (3.6). Notice that the decoupled 
feature essentially comes from our assumption of $A_{+}=A_{-}=A$ and $\nu_{+}=\nu_{-}=\nu$ in (1.1). The linearized homogeneous system of (3.4) reads

$$
\left\{\begin{array}{l}
\partial_{t} \rho_{1}+\nabla \cdot u_{1}=0, \\
\partial_{t} u_{1}+u_{1}+E+\gamma \nabla \rho_{1}=0, \\
\partial_{t} E-\nabla \times B-2 u_{1}=0, \\
\partial_{t} B+\nabla \times E=0, \\
\frac{1}{2} \nabla \cdot E=-\rho_{1}, \quad \nabla \cdot B=0, \quad t>0, x \in \mathbb{R}^{3},
\end{array}\right.
$$

with initial data

$$
\left.U_{1}\right|_{t=0}=U_{1,0}:=\left[\rho_{1,0}, u_{1,0}, E_{0}, B_{0}\right], \quad x \in \mathbb{R}^{3},
$$

satisfying the compatibility conditions

$$
\frac{1}{2} \nabla \cdot E=-\rho_{1,0}, \quad \nabla \cdot B_{0}=0 .
$$

And the linearized homogeneous equations of (3.6) become

$$
\left\{\begin{array}{l}
\partial_{t} \rho_{2}+\nabla \cdot u_{2}=0, \\
\partial_{t} u_{2}+u_{2}+\gamma \nabla \rho_{2}=0, \quad t>0, x \in \mathbb{R}^{3},
\end{array}\right.
$$

with initial data

$$
\left.U_{2}\right|_{t=0}=U_{2,0}:=\left[\rho_{2,0}, u_{2,0}\right], \quad x \in \mathbb{R}^{3} .
$$

Here $\left[\rho_{2,0}, u_{2,0}\right]$ is given from $\left[\rho_{+0}, \rho_{-0}, u_{+0}, u_{0}\right]$ according to the transform (3.5).

From now on, we always denote $U_{1}=\left[\rho_{1}, u_{1}, E, B\right]$ as the solution to the linearized homogeneous system (3.10), and $U_{2}=\left[\rho_{2}, u_{2}\right]$ as the one to (3.13). And since smooth solutions to the nonlinear system (2.2) and (3.1) are equivalent, time-decay properties of the solution to (3.1) can be directly applied to (2.2).

First of all, for the linearized equations (3.10)-(3.12), the $L^{p}-L^{q}$ time decay property was proved in [6]. We only list some special $L^{p}-L^{q}$ time decay inequalities in the following

Proposition 3.1. Suppose $U_{1}(t)=e^{t L} U_{1,0}$ is the solution to the Cauchy problem (3.10)(3.11) with the initial data $U_{1,0}=\left[\rho_{1,0}, u_{1,0}, E_{0}, B_{0}\right]$ satisfying (3.12). Then $U_{1}=\left[\rho_{1}, u_{1}, E, B\right]$ satisfies the following time-decay property:

$$
\left\{\begin{array}{l}
\left\|\rho_{1}(t)\right\| \leq C e^{-\frac{t}{2}}\left\|\left[\rho_{1,0}, u_{1,0}\right]\right\| \\
\left\|u_{1}(t)\right\| \leq C e^{-\frac{t}{2}}\left\|\rho_{1,0}\right\|+C(1+t)^{-\frac{5}{4}}\left\|\left[u_{1,0}, E_{0}, B_{0}\right]\right\|_{L^{1} \cap \dot{H}^{2}}, \\
\|E(t)\| \leq C(1+t)^{-\frac{5}{4}}\left\|\left[u_{1,0}, E_{0}, B_{0}\right]\right\|_{L^{1} \cap \dot{H}^{3}}, \\
\|B(t)\| \leq C(1+t)^{-\frac{3}{4}}\left\|\left[u_{1,0}, E_{0}, B_{0}\right]\right\|_{L^{1} \cap \dot{H}^{2}},
\end{array}\right.
$$

and

$$
\left\{\begin{array}{l}
\left\|\rho_{1}(t)\right\|_{\infty} \leq C e^{-\frac{t}{2}}\left\|\left[\rho_{1,0}, u_{1,0}\right]\right\|_{L^{2} \cap \dot{H}^{2}}, \\
\left\|u_{1}(t)\right\|_{\infty} \leq C e^{-\frac{t}{2}}\left\|\rho_{1,0}\right\|_{L^{2} \cap \dot{H}^{2}}+C(1+t)^{-2}\left\|\left[u_{1,0}, E_{0}, B_{0}\right]\right\|_{L^{1} \cap \dot{H}^{5}}, \\
\|E(t)\|_{\infty} \leq C(1+t)^{-2}\left\|\left[u_{1,0}, E_{0}, B_{0}\right]\right\|_{L^{1} \cap \dot{H}^{6}}, \\
\|B(t)\|_{\infty} \leq C(1+t)^{-\frac{3}{2}}\left\|\left[u_{1,0}, E_{0}, B_{0}\right]\right\|_{L^{1} \cap \dot{H}^{5}},
\end{array}\right.
$$


and moreover,

$$
\left\{\begin{array}{l}
\|\nabla B(t)\| \leq C(1+t)^{-\frac{5}{4}}\left\|\left[u_{1,0}, E_{0}, B_{0}\right]\right\|_{L^{1} \cap \dot{H}^{4}}, \\
\left\|\nabla^{N}[E(t), B(t)]\right\| \leq C(1+t)^{-\frac{5}{4}}\left\|\left[u_{1,0}, E_{0}, B_{0}\right]\right\|_{L^{1} \cap \dot{H}^{N+3}} .
\end{array}\right.
$$

As we mention before, the time-decay property of the damped Euler system (3.13) was studied in [18]. The rest of this section is to get the refined time-decay property by exploring the explicit solution to the Cauchy problem (3.13)-(3.14). In fact, compared with the result in $[18$, Lemma 6.1], it will be shown that the momentum component has an extra time-decay $(1+t)^{-1 / 2}$ in $L^{2}$.

3.1. Representation of solutions for (3.13) and (3.14). We first find the explicit representation of the Fourier transform of the solution $U_{2}=\left[\rho_{2}, u_{2}\right]=e^{t L} U_{2,0}$ to the Cauchy problem (3.13)-(3.14). For an integrable function $f: \mathbb{R}^{3} \rightarrow \mathbb{R}$, its Fourier transform is defined by

$$
\hat{f}(k)=\int_{\mathbb{R}^{3}} e^{-i x \cdot k} f(x) d x, \quad x \cdot k:=\sum_{j=1}^{3} x_{j} k_{j}, \quad k \in \mathbb{R}^{3},
$$

where $i=\sqrt{-1} \in \mathbb{C}$ is the imaginary unit.

Taking the time derivative for the first equation of (3.13), and using the second equation to replace $\partial_{t} u_{2}$ we have

$$
\partial_{t t} \rho_{2}+\partial_{t} \rho_{2}-\gamma \triangle \rho_{2}=0
$$

Initial data is given by

$$
\left.\rho_{2}\right|_{t=0}=\rho_{2,0}=\frac{\rho_{+0}+\rho_{-0}}{2},\left.\partial_{t} \rho_{2}\right|_{t=0}=-\nabla \cdot u_{2,0} .
$$

By taking the Fourier transform of (3.18)-(3.19), we get the second order ODE as

$$
\left\{\begin{array}{l}
\partial_{t t} \hat{\rho}_{2}+\partial_{t} \hat{\rho}_{2}+\gamma|k|^{2} \hat{\rho}_{2}=0 \\
\left.\hat{\rho}_{2}\right|_{t=0}=\hat{\rho}_{2,0} \\
\left.\partial_{t} \hat{\rho}_{2}\right|_{t=0}=-i k \cdot \hat{u}_{2,0}
\end{array}\right.
$$

It is straightforward to obtain

$$
\hat{\rho}_{2}=\hat{\rho}_{2,0} \frac{\chi_{+} e^{\chi_{-} t}-\chi_{-} e^{\chi_{+} t}}{\chi_{+}-\chi_{-}}-i k \cdot \hat{u}_{2,0} \frac{e^{\chi_{+} t}-e^{\chi_{-} t}}{\chi_{+}-\chi_{-}},
$$

where $\chi_{ \pm}=-\frac{1}{2} \pm \frac{1}{2} \sqrt{1-4 \gamma\left|k^{2}\right|}$ are the roots of the characteristic equation $\chi^{2}+\chi+\gamma|k|^{2}=0$. Similarly, taking the time derivative for the second equation of (3.13), and using the first equation to replace $\partial_{t} \rho_{2}$ we have

$$
\partial_{t t} u_{2}+\partial_{t} u_{2}-\gamma \nabla\left(\nabla \cdot u_{2}\right)=0 .
$$

Further taking the divergence, one has

$$
\partial_{t t}\left(\nabla \cdot u_{2}\right)+\partial_{t}\left(\nabla \cdot u_{2}\right)-\gamma \Delta\left(\nabla \cdot u_{2}\right)=0 .
$$

Notice

$$
\begin{gathered}
\left.\nabla \cdot u_{2}\right|_{t=0}=\nabla \cdot u_{2,0}, \\
\left.\partial_{t} \nabla \cdot u_{2}\right|_{t=0}=-\nabla \cdot u_{2,0}-\gamma \Delta \rho_{2,0} .
\end{gathered}
$$


Here and in the sequel we set $\tilde{k}=k /|k|$ for $|k| \neq 0$. By taking the Fourier transform of (3.20), (3.21) and (3.22), we get the second order ODE as

$$
\left\{\begin{array}{l}
\partial_{t t}\left(\tilde{k} \cdot \hat{u}_{2}\right)+\partial_{t}\left(\tilde{k} \cdot \hat{u}_{2}\right)+\gamma|k|^{2}\left(\tilde{k} \cdot \hat{u}_{2}\right)=0, \\
\left.\left(\tilde{k} \cdot \hat{u}_{2}\right)\right|_{t=0}=\tilde{k} \cdot \hat{u}_{2,0} \\
\left.\partial_{t}\left(\tilde{k} \cdot \hat{u}_{2}\right)\right|_{t=0}=-\tilde{k} \cdot \hat{u}_{2,0}-i \gamma|k| \hat{\rho}_{2,0} .
\end{array}\right.
$$

Therefore,

$$
\tilde{k} \cdot \hat{u}_{2}=\frac{\chi_{+} e^{\chi_{+} t}-\chi_{-} e^{\chi_{-} t}}{\chi_{+}-\chi_{-}} \tilde{k} \cdot \hat{u}_{2,0}+\frac{e^{\chi_{+} t}-e^{\chi_{-} t}}{\chi_{+}-\chi_{-}}\left[-i \gamma|k| \hat{\rho}_{2,0}\right] .
$$

Here we have used the fact $\chi_{+}+1=-\chi_{-}$and $\chi_{-}+1=-\chi_{+}$.

Moreover, by taking the curl for the equation of $\partial_{t} u_{2}$ and in terms of the Fourier transformation in $x$, one has

$$
\partial_{t}\left(\tilde{k} \times \hat{u}_{2}\right)+\left(\tilde{k} \times \hat{u}_{2}\right)=0 \text {. }
$$

Initial data is given as

$$
\left.\left(\tilde{k} \times \hat{u}_{2}\right)\right|_{t=0}=\tilde{k} \times \hat{u}_{2,0} .
$$

By solving the initial value problem (3.23) and (3.24), one has

$$
\tilde{k} \times \hat{u}_{2}=e^{-t}\left(\tilde{k} \times \hat{u}_{2,0}\right) .
$$

For $t \geq 0$ and $k \in \mathbb{R}^{3}$ with $|k| \neq 0$, one has the decomposition $\hat{u}_{2}=\tilde{k} \tilde{k} \cdot \hat{u}_{2}-\tilde{k} \times\left(\tilde{k} \times \hat{u}_{2}\right)$ as in $[6]$.

$$
\begin{aligned}
\hat{u}_{2}= & \frac{\chi_{+} e^{\chi_{+} t}-\chi_{-} e^{\chi_{-} t}}{\chi_{+}-\chi_{-}} \tilde{k} \cdot \hat{u}_{2,0} \\
& +\frac{e^{\chi_{+} t}-e^{\chi_{-} t}}{\chi_{+}-\chi_{-}}\left[-i \gamma k \hat{\rho}_{2,0}\right]-e^{-t} \tilde{k} \times\left(\tilde{k} \times \hat{u}_{2,0}\right) .
\end{aligned}
$$

Now, let us summarize the above computations on the explicit representation of Fourier transform of the solution $U_{2}=\left[\rho_{2}, u_{2}\right]$.

$$
\left(\begin{array}{c}
\hat{\rho}_{2}(t, k) \\
\hat{u}_{2}(t, k)
\end{array}\right)=\hat{G}(t, k)\left(\begin{array}{c}
\hat{\rho}_{2}(0, k) \\
\hat{u}_{2}(0, k)
\end{array}\right)
$$

with

$$
\hat{G}(t, k)=\left(\begin{array}{cc}
\frac{\chi_{+} e^{\chi_{-} t}-\chi_{-} e^{\chi_{+} t}}{\chi_{+}-\chi_{-}} & \frac{e^{\chi_{+} t}-e^{\chi_{-}}}{\chi_{+}-\chi_{-}}\left(-i k^{T}\right) \\
\frac{e^{\chi_{+}{ }^{-}-e^{\chi_{-}}}}{\chi_{+}-\chi_{-}}(-i \gamma k) & e^{-t}\left(\mathbf{I}_{3}-\frac{k \otimes k}{|k|^{2}}\right)+\frac{\chi_{+} e^{\chi_{+}}-\chi_{-} e_{-} t}{\chi_{+}-\chi_{-}} \frac{k \otimes k}{|k|^{2}}
\end{array}\right),
$$

where the superscript $T$ denotes the transpose of the vector $k$.

3.2. Refined $L^{p}-L^{q}$ time-decay property. In this subsection, we use (3.25) to obtain some refined $L^{p}-L^{q}$ time-decay property for $U_{2}=\left[\rho_{2}, u_{2}\right]$. For that, we first find the timefrequency pointwise estimates on $\hat{\rho}_{2}, \hat{u}_{2}$ in the following lemma; see [10] for the similar study of the linearized Navier-Stokes system.

Lemma 3.1. Let $U_{2}=\left[\rho_{2}, u_{2}\right]$ be the solution to the linearized homogeneous system (3.13) with initial data $U_{2,0}=\left[\rho_{2,0}, u_{2,0}\right]$. Then, there exist constants $\varepsilon>0, \lambda>0, C>0$ such that for all $t>0,|k| \leq \varepsilon$,

$$
\begin{aligned}
\left|\hat{\rho}_{2}(t, k)\right| \leq & C\left(|k|^{2} e^{-\lambda t}+e^{-\lambda|k|^{2} t}\right)\left|\hat{\rho}_{2,0}(k)\right| \\
& +C\left(|k| e^{-\lambda|k|^{2} t}+|k| e^{-\lambda t}\right)\left|\hat{u}_{2,0}(k)\right|,
\end{aligned}
$$




$$
\begin{aligned}
\left|\hat{u}_{2}(t, k)\right| \leq & C\left(|k| e^{-\lambda|k|^{2} t}+|k| e^{-\lambda t}\right)\left|\hat{\rho}_{2,0}(k)\right| \\
& +C\left(|k|^{2} e^{-\lambda|k|^{2} t}+e^{-\lambda t}\right)\left|\hat{u}_{2,0}(k)\right|,
\end{aligned}
$$

and for all $t>0,|k| \geq \varepsilon$,

$$
\begin{aligned}
& \left|\hat{\rho}_{2}(t, k)\right| \leq C e^{-\lambda t}\left|\left[\hat{\rho}_{2,0}(k), \hat{u}_{2,0}(k)\right]\right|, \\
& \left|\hat{u}_{2}(t, k)\right| \leq C e^{-\lambda t}\left|\left[\hat{\rho}_{2,0}(k), \hat{u}_{2,0}(k)\right]\right| .
\end{aligned}
$$

Proof. In order to get the upper bound of $\hat{\rho}_{2}(t, k)$ and $\hat{u}_{2}(t, k)$, we have to estimate $\hat{G}_{11}, \hat{G}_{12}$, $\hat{G}_{21}$ and $\hat{G}_{22}$. Here we denote

$$
\begin{aligned}
& \hat{G}_{11}=\frac{\chi_{+} e^{\chi_{-} t}-\chi_{-} e^{\chi_{+} t}}{\chi_{+}-\chi_{-}}, \quad \hat{G}_{12}=\frac{e^{\chi_{+} t}-e^{\chi_{-} t}}{\chi_{+}-\chi_{-}}\left(-i k^{T}\right) \\
& \hat{G}_{21}=\frac{e^{\chi_{+} t}-e^{\chi_{-} t}}{\chi_{+}-\chi_{-}}(-i \gamma k), \quad \hat{G}_{22}=e^{-t}\left(\mathbf{I}_{3}-\frac{k \otimes k}{|k|^{2}}\right)+\frac{\chi_{+} e^{\chi_{+} t}-\chi_{-} e^{\chi_{-} t}}{\chi_{+}-\chi_{-}} \frac{k \otimes k}{|k|^{2}} .
\end{aligned}
$$

If $1-4 \gamma\left|k^{2}\right| \geq 0$, then $\chi_{ \pm}=-\frac{1}{2} \pm \frac{1}{2} \sqrt{1-4 \gamma|k|^{2}}$ are real. It is straightforward to obtain

$$
\begin{aligned}
& \chi_{+}=-O(1)|k|^{2}, \chi_{-}=-1+O(1)|k|^{2}, \\
& \chi_{+}-\chi_{-}=\sqrt{1-4 \gamma|k|^{2}}=O(1),
\end{aligned}
$$

as $|k| \rightarrow 0$. And on the other hand, if $1-4 \gamma\left|k^{2}\right|<0$, then $\chi_{ \pm}=-\frac{1}{2} \pm \frac{1}{2} i \sqrt{4 \gamma|k|^{2}-1}$ are complex conjugate. Moreover, one has

$$
\begin{aligned}
& \left|\chi_{ \pm}\right|=O(1)|k| \\
& \chi_{+}-\chi_{-}=i \sqrt{4 \gamma|k|^{2}-1}=i O(1)|k|,
\end{aligned}
$$

as $|k| \rightarrow \infty$. Then, there exists $\varepsilon \leq \sqrt{\frac{1}{4 \gamma}} \leq R$, with $0<\varepsilon \ll 1 \ll R<\infty$ such that one can estimate $\hat{G}$ as follows:

$$
\begin{aligned}
\left|\hat{G}_{11}\right| & \leq C|k|^{2} e^{-\lambda t}+C e^{-\lambda|k|^{2} t} \\
\left|\hat{G}_{12}\right| & +\left|\hat{G}_{21}\right| \leq C|k|\left(e^{-\lambda t}+e^{-\lambda|k|^{2} t}\right) \\
\left|\hat{G}_{22}\right| & \leq C e^{-t}+C|k|^{2} e^{-\lambda|k|^{2} t}+C e^{-\lambda t} \\
& \leq C|k|^{2} e^{-\lambda|k|^{2} t}+C e^{-\lambda t}
\end{aligned}
$$

as $|k| \leq \varepsilon$, and

$$
\begin{aligned}
& \left|\hat{G}_{11}\right| \leq C e^{-\frac{1}{2} t} \leq C e^{-\lambda t} \\
& \left|\hat{G}_{12}\right|+\left|\hat{G}_{21}\right| \leq C e^{-\frac{1}{2} t} \leq C e^{-\lambda t} \\
& \left|\hat{G}_{22}\right| \leq C e^{-t}+e^{-\frac{1}{2} t} \leq C e^{-\lambda t}
\end{aligned}
$$

as $|k| \geq R$.

In what follows we estimate only $\hat{G}_{12}$ over $\varepsilon \leq|k| \leq R$. When $|k| \leq \frac{1}{\sqrt{4 \gamma}}$,

$$
\lim _{|k| \rightarrow \sqrt{\frac{1}{4 \gamma}}} \frac{e^{\chi_{+} t}-e^{\chi_{-} t}}{\chi_{+}-\chi_{-}}=t e^{-\frac{1}{2} t} \leq C e^{-\lambda t} .
$$


When $|k| \geq \frac{1}{\sqrt{4 \gamma}}$

$$
\frac{e^{\chi+t}-e^{\chi-t}}{\chi_{+}-\chi_{-}}=\frac{2 e^{-\frac{1}{2} t} \sin \left(\frac{1}{2} \sqrt{4 \gamma|k|^{2}-1} t\right)}{\sqrt{4 \gamma|k|^{2}-1}}
$$

and

$$
\lim _{|k| \rightarrow \sqrt{\frac{1}{4 \gamma}}} \frac{2 e^{-\frac{1}{2} t} \sin \left(\frac{1}{2} \sqrt{4 \gamma|k|^{2}-1} t\right)}{\sqrt{4 \gamma|k|^{2}-1}}=t e^{-\frac{1}{2} t} \leq C e^{-\lambda t} .
$$

Then there exists $\delta>0$, if ||$k\left|-\frac{1}{\sqrt{4 \gamma}}\right| \leq \delta$, one has

$$
\left|\frac{e^{\chi_{+} t}-e^{\chi_{-} t}}{\chi_{+}-\chi_{-}}\right| \leq C e^{-\lambda t}, \quad\left|\frac{e^{\chi_{+} t}-e^{\chi_{-} t}}{\chi_{+}-\chi_{-}}\left(-i k^{T}\right)\right| \leq C e^{-\lambda t} .
$$

Next, let's consider $\hat{G}_{12}$ over $\left\{\varepsilon \leq|k| \leq R,|| k\left|-\frac{1}{\sqrt{4 \gamma}}\right| \geq \delta\right\}$. Notice that in this domain,

$$
\left|\frac{e^{\chi_{+} t}-e^{\chi_{-} t}}{\chi_{+}-\chi_{-}}\left(-i k^{T}\right)\right| \leq C e^{-\lambda t},
$$

where the fact that

$$
\begin{cases}\chi_{ \pm}<0 & \text { whenever } \chi_{ \pm} \text {real } \\ \mathfrak{R} \chi_{ \pm}=-\frac{1}{2} & \text { whenever } \chi_{ \pm} \text {non-real and conjugate }\end{cases}
$$

has been used. Therefore, in the completely same way, we can get

$$
\left|\hat{G}_{11}\right|+\left|\hat{G}_{21}\right|+\left|\hat{G}_{22}\right| \leq C e^{-\lambda t},
$$

over $\varepsilon \leq|k| \leq R$. In summary,

$$
\begin{aligned}
\left|\hat{G}_{11}\right| & \leq C|k|^{2} e^{-\lambda t}+C e^{-\lambda|k|^{2} t} \\
\left|\hat{G}_{12}\right| & +\left|\hat{G}_{21}\right| \leq C|k|\left(e^{-\lambda t}+e^{-\lambda|k|^{2} t}\right) \\
\left|\hat{G}_{22}\right| & \leq C e^{-t}+|k|^{2} e^{-\lambda|k|^{2} t}+e^{-\lambda t} \\
& \leq C|k|^{2} e^{-\lambda|k|^{2} t}+C e^{-\lambda t}
\end{aligned}
$$

as $|k| \leq \varepsilon$, and

$$
\left|\hat{G}_{i j}\right| \leq C e^{-\lambda t}, \quad 1 \leq i, j \leq 2,
$$

as $|k| \geq \varepsilon$.

Now, in terms of $(3.30)$, we can estimate $\hat{\rho}_{2}(t, k), \hat{u}_{2}(t, k)$ as

$$
\begin{aligned}
\left|\hat{\rho}_{2}(t, k)\right|= & \left|\hat{G}_{11} \hat{\rho}_{2,0}(k)+\hat{G}_{12} \hat{u}_{2,0}(k)\right| \\
\leq & \left|\hat{G}_{11}\right|\left|\hat{\rho}_{2,0}(k)\right|+\left|\hat{G}_{12}\right|\left|\hat{u}_{2,0}(k)\right| \\
\leq & C\left(|k|^{2} e^{-\lambda t}+e^{-\lambda|k|^{2} t}\right)\left|\hat{\rho}_{2,0}(k)\right| \\
& +C\left(|k| e^{-\lambda|k|^{2} t}+|k| e^{-\lambda t}\right)\left|\hat{u}_{2,0}(k)\right|, \\
\left|\hat{u}_{2}(t, k)\right|= & \left|\hat{G}_{21} \hat{\rho}_{2,0}(k)+\hat{G}_{22} \hat{u}_{2,0}(k)\right| \\
\leq & \left|\hat{G}_{21}\right|\left|\hat{\rho}_{2,0}(k)\right|+\left|\hat{G}_{22}\right|\left|\hat{u}_{2,0}(k)\right| \\
\leq & C\left(|k| e^{-\lambda|k|^{2} t}+|k| e^{-\lambda t}\right)\left|\hat{\rho}_{2,0}(k)\right| \\
& +C\left(|k|^{2} e^{-\lambda|k|^{2} t}+e^{-\lambda t}\right)\left|\hat{u}_{2,0}(k)\right|,
\end{aligned}
$$


for $|k| \leq \varepsilon$, which prove (3.26) and (3.27). Finally, (3.28) and (3.29) directly follow from (3.31). This completes the proof of Lemma 3.1.

Based on Lemma 3.1, it is standard to obtain the following time-decay property for each component of the solution $\left[\rho_{2}, u_{2}\right]$; the details of proof are omitted for simplicity.

Theorem 3.1. Let $1 \leq p, r \leq 2 \leq q \leq \infty$, and let $m \geq 1$ be an integer. Suppose that $U_{2}(t)=e^{t L} U_{2,0}$ is the solution to the Cauchy problem (3.13)-(3.14). Then $U_{2}=\left[\rho_{2}, u_{2}\right]$ satisfies

$$
\begin{aligned}
\left\|\nabla^{m} \rho_{2}(t)\right\|_{L_{x}^{q}} \leq C(1+t)^{-\frac{3}{2}\left(\frac{1}{p}-\frac{1}{q}\right)-\frac{m}{2}}\left\|\left[\rho_{2,0}, u_{2,0}\right]\right\|_{L^{p}} \\
+C e^{-\lambda t}\left\|\nabla^{m+\left[3\left(\frac{1}{r}-\frac{1}{q}\right)\right]_{+}}\left[\rho_{2,0}, u_{2,0}\right]\right\|_{L^{r}}, \\
\left\|\nabla^{m} u_{2}(t)\right\|_{L_{x}^{q}} \leq C(1+t)^{-\frac{3}{2}\left(\frac{1}{p}-\frac{1}{q}\right)-\frac{m+1}{2}}\left\|\left[\rho_{2,0}, u_{2,0}\right]\right\|_{L^{p}} \\
+C e^{-\lambda t}\left\|\nabla^{m+\left[3\left(\frac{1}{r}-\frac{1}{q}\right)\right]_{+}}\left[\rho_{2,0}, u_{2,0}\right]\right\|_{L^{r}},
\end{aligned}
$$

for any $t \geq 0$, where $C=C(m, p, r, q)$ and $\left[3\left(\frac{1}{r}-\frac{1}{q}\right)\right]_{+}$is defined as

$$
\left[3\left(\frac{1}{r}-\frac{1}{q}\right)\right]_{+}= \begin{cases}0, & \text { if } \ell \text { is integer and } r=q=2, \\ {\left[3\left(\frac{1}{r}-\frac{1}{q}\right)\right]_{-}+1,} & \text { otherwise }\end{cases}
$$

where $[\cdot]_{-}$denotes the integer part of the argument.

For later use, from Theorem 3.1, let us list some special cases in the following

Corollary 3.1. Suppose $U_{2}(t)=e^{t L} U_{2,0}$ is the solution to the Cauchy problem (3.13) with initial data (3.14). Then $U_{2}=\left[\rho_{2}, u_{2}\right]$ satisfies

$$
\begin{gathered}
\left\{\begin{array}{l}
\left\|\rho_{2}(t)\right\| \leq C(1+t)^{-\frac{3}{4}}\left\|\left[\rho_{2,0}, u_{2,0}\right]\right\|_{L^{1}}+C e^{-\lambda t}\left\|\left[\rho_{2,0}, u_{2,0}\right]\right\|, \\
\left\|u_{2}(t)\right\| \leq C(1+t)^{-\frac{5}{4}}\left\|\left[\rho_{2,0}, u_{2,0}\right]\right\|_{L^{1}}+C e^{-\lambda t}\left\|\left[\rho_{2,0}, u_{2,0}\right]\right\|,
\end{array}\right. \\
\left\{\begin{array}{l}
\left\|\nabla \rho_{2}(t)\right\| \leq C(1+t)^{-\frac{5}{4}}\left\|\left[\rho_{2,0}, u_{2,0}\right]\right\|_{L^{1}}+C e^{-\lambda t}\left\|\nabla\left[\rho_{2,0}, u_{2,0}\right]\right\|, \\
\left\|\nabla u_{2}(t)\right\| \leq C(1+t)^{-\frac{7}{4}}\left\|\left[\rho_{2,0}, u_{2,0}\right]\right\|_{L^{1}}+C e^{-\lambda t}\left\|\nabla\left[\rho_{2,0}, u_{2,0}\right]\right\|,
\end{array}\right. \\
\left\{\begin{array}{l}
\left\|\rho_{2}(t)\right\|_{\infty} \leq C(1+t)^{-\frac{3}{2}}\left\|\left[\rho_{2,0}, u_{2,0}\right]\right\|_{L^{1}}+C e^{-\lambda t}\left\|\nabla^{2}\left[\rho_{2,0}, u_{2,0}\right]\right\|, \\
\left\|u_{2}(t)\right\|_{\infty} \leq C(1+t)^{-2}\left\|\left[\rho_{2,0}, u_{2,0}\right]\right\|_{L^{1}}+C e^{-\lambda t}\left\|\nabla^{2}\left[\rho_{2,0}, u_{2,0}\right]\right\| .
\end{array}\right.
\end{gathered}
$$

\section{DeCay in time for the NON-LINEAR System}

4.1. Time rate for full instant energy functional. In this subsection, we shall prove (2.12) in Proposition 2.2. The main idea follows from [7] for introducing a general approach of the combination of energy estimates and spectral analysis. First of all, from Theorem 2.1, one has

Lemma 4.1. Let $V=\left[\sigma_{ \pm}, v_{ \pm}, \tilde{E}, \tilde{B}\right]$ be the solution to the the Cauchy problem (2.2)-(2.3) with initial data $V_{0}=\left[\sigma_{ \pm 0}, v_{ \pm 0}, \tilde{E}_{0}, \tilde{B}_{0}\right]$ satisfying $(2.4)$. Then, if $\mathcal{E}_{N}\left(V_{0}\right)$ is sufficiently small,

$$
\frac{d}{d t} \mathcal{E}_{N}(V(t))+\lambda \mathcal{D}_{N}(V(t)) \leq 0
$$

holds for any $t>0$, where $\mathcal{E}_{N}(V(t)), \mathcal{D}_{N}(V(t))$ are in the form of $(2.5)$ and $(2.7)$, respectively. 
Now, we proceed by making the time-weighted estimate and iteration for the Lyapunov inequality (4.1). Let $\ell \geq 0$. Multiplying (4.1) by $(1+t)^{\ell}$ and taking integration over $[0, t]$, one gets

$$
\begin{aligned}
& (1+t)^{\ell} \mathcal{E}_{N}(V(t))+\lambda \int_{0}^{t}(1+s)^{\ell} \mathcal{D}_{N}(V(s)) d s \\
\leq & \mathcal{E}_{N}\left(V_{0}\right)+\ell \int_{0}^{t}(1+s)^{\ell-1} \mathcal{E}_{N}(V(s)) d s .
\end{aligned}
$$

Noticing

$$
\mathcal{E}_{N}(V(t)) \leq C\left(D_{N+1}(V(t))+\|\tilde{B}\|^{2}+\left\|\sigma_{+}+\sigma_{-}\right\|^{2}\right)
$$

it follows that

$$
\begin{aligned}
& (1+t)^{\ell} \mathcal{E}_{N}(V(t))+\lambda \int_{0}^{t}(1+s)^{\ell} \mathcal{D}_{N}(V(s)) d s \\
\leq & \mathcal{E}_{N}\left(V_{0}\right)+C \ell \int_{0}^{t}(1+s)^{\ell-1}\left(\|\tilde{B}\|^{2}+\left\|\sigma_{+}+\sigma_{-}\right\|^{2}\right) d s \\
& +C \ell \int_{0}^{t}(1+s)^{\ell-1} \mathcal{D}_{N+1}(V(s)) d s .
\end{aligned}
$$

Similarly, it holds that

$$
\begin{aligned}
& (1+t)^{\ell-1} \mathcal{E}_{N+1}(V(t))+\lambda \int_{0}^{t}(1+s)^{\ell-1} \mathcal{D}_{N+1}(V(s)) d s \\
\leq & \mathcal{E}_{N+1}\left(V_{0}\right)+C(\ell-1) \int_{0}^{t}(1+s)^{\ell-2}\left(\|\tilde{B}\|^{2}+\left\|\sigma_{+}+\sigma_{-}\right\|^{2}\right) d s \\
& +C(\ell-1) \int_{0}^{t}(1+s)^{\ell-2} \mathcal{D}_{N+2}(V(s)) d s,
\end{aligned}
$$

and

$$
\mathcal{E}_{N+2}(V(t))+\lambda \int_{0}^{t} \mathcal{D}_{N+2}(V(s)) d s \leq \mathcal{E}_{N+2}\left(V_{0}\right) .
$$

Then, for $1<\ell<2$, by iterating the above estimates, one has

$$
\begin{aligned}
& (1+t)^{\ell} \mathcal{E}_{N}(V(t))+\lambda \int_{0}^{t}(1+s)^{\ell} \mathcal{D}_{N}(V(s)) d s \\
\leq & C \mathcal{E}_{N+2}\left(V_{0}\right)+C \int_{0}^{t}(1+s)^{\ell-1}\left(\|\tilde{B}\|^{2}+\left\|\sigma_{+}+\sigma_{-}\right\|^{2}\right) d s .
\end{aligned}
$$

For this time, to estimate the time integral term on the r.h.s. of (4.2), let's define

$$
\mathcal{E}_{N, \infty}(V(t))=\sup _{0 \leq s \leq t}(1+s)^{\frac{3}{2}} \mathcal{E}_{N}(V(s)) .
$$

Lemma 4.2. For any $t \geq 0$, it holds that

$$
\begin{aligned}
\|\tilde{B}\|^{2}+\left\|\sigma_{+}+\sigma_{-}\right\|^{2} \leq C(1+t)^{-\frac{3}{2}}\left(\mathcal{E}_{N, \infty}^{2}(V(t))\right. & +\left\|\left[\sigma_{ \pm 0}, v_{ \pm 0}\right]\right\|_{L^{1} \cap L^{2}}^{2} \\
& \left.+\left\|\left[v_{ \pm 0}, \tilde{E}_{0}, \tilde{B}_{0}\right]\right\|_{L^{1} \cap \dot{H}^{2}}^{2}\right) .
\end{aligned}
$$

Proof. By applying the fourth linear estimate on $B$ in (3.15) and the first linear estimate on $\rho_{2}$ in (3.32) to the mild form (3.8) and (3.9), respectively, one has

$$
\begin{aligned}
\|B(t)\| \leq C(1+t)^{-\frac{3}{4}} \| & {\left[u_{ \pm 0}, E_{0}, B_{0}\right] \|_{L^{1} \cap \dot{H}^{2}} } \\
& +C \int_{0}^{t}(1+t-s)^{-\frac{3}{4}}\left\|\left[g_{2}^{+}(s)-g_{2}^{-}(s), g_{3}^{+}(s)-g_{3}^{+}(s)\right]\right\|_{L^{1} \cap \dot{H}^{2}} d s,
\end{aligned}
$$




$$
\begin{aligned}
\left\|\left[\rho_{+}(t)+\rho_{-}(t)\right]\right\| \leq & C\left\|\rho_{2}\right\| \leq C e^{-\lambda t}\left\|\left[\rho_{ \pm 0}, u_{ \pm 0}\right]\right\|+C(1+t)^{-\frac{3}{4}}\left\|\left[\rho_{ \pm 0}, u_{ \pm 0}\right]\right\|_{L^{1}} \\
& +C \int_{0}^{t} e^{-\lambda(t-s)}\left\|\left[g_{1}^{+}(s)+g_{1}^{-}(s), g_{2}^{+}(s)+g_{2}^{-}(s)\right]\right\| d s \\
& +C \int_{0}^{t}(1+t-s)^{-\frac{3}{4}}\left\|\left[g_{1}^{+}(s)+g_{1}^{-}(s), g_{2}^{+}(s)+g_{2}^{-}(s)\right]\right\|_{L^{1}} d s .
\end{aligned}
$$

Here, the definition for $\rho_{1}, u_{1}$ and $\rho_{2}, u_{2}$ in (3.3) and (3.5) has been used.

Recall the definition (3.7) of $g_{1}^{+}, g_{1}^{-}, g_{2}^{+} g_{2}^{-}$and $g_{3}^{+}, g_{3}^{-}$. It is straightforward to verify that for any $0 \leq s \leq t$,

$$
\begin{aligned}
& \left\|\left[g_{2}^{+}(s)-g_{2}^{-}(s), g_{3}^{+}(s)-g_{3}^{+}(s)\right]\right\|_{L^{1} \cap \dot{H}^{2}} \leq C \mathcal{E}_{N}(U(s)), \\
& \left\|\left[g_{1}^{+}(s)+g_{1}^{-}(s), g_{2}^{+}(s)+g_{2}^{-}(s)\right]\right\|_{L^{1} \cap L^{2}} \leq C \mathcal{E}_{N}(U(s)) .
\end{aligned}
$$

Notice that $\mathcal{E}_{N}(U(s)) \leq C \mathcal{E}_{N}(V(\sqrt{\gamma} s))$. From (4.3), for any $0 \leq s \leq t$,

$$
\mathcal{E}_{N}(V(\sqrt{\gamma} s)) \leq(1+\sqrt{\gamma} s)^{-\frac{3}{2}} \mathcal{E}_{N, \infty}(V(\sqrt{\gamma} t)) .
$$

Then, it follows that for $0 \leq s \leq t$,

$$
\begin{gathered}
\left\|\left[g_{2}^{+}(s)-g_{2}^{-}(s), g_{3}^{+}(s)-g_{3}^{+}(s)\right]\right\|_{L^{1} \cap \dot{H}^{2}} \leq C(1+\sqrt{\gamma} s)^{-\frac{3}{2}} \mathcal{E}_{N, \infty}(V(\sqrt{\gamma} t), \\
\left\|\left[g_{1}^{+}(s)+g_{1}^{-}(s), g_{2}^{+}(s)+g_{2}^{-}(s)\right]\right\|_{L^{1} \cap L^{2}} \leq C(1+\sqrt{\gamma} s)^{-\frac{3}{2}} \mathcal{E}_{N, \infty}(V(\sqrt{\gamma} t) .
\end{gathered}
$$

Putting the above two inequalities into (4.5) and (4.6) respectively gives

$$
\begin{gathered}
\|B(t)\| \leq C(1+t)^{-\frac{3}{4}}\left(\left\|\left[u_{ \pm 0}, E_{0}, B_{0}\right]\right\|_{L^{1} \cap \dot{H}^{2}}+\mathcal{E}_{N, \infty}(V(\sqrt{\gamma} t))\right), \\
\left\|\rho_{+}(t)+\rho_{-}(t)\right\| \leq C(1+t)^{-\frac{3}{4}}\left(\left\|\left[\rho_{ \pm 0}, u_{ \pm 0}\right]\right\|_{L^{1} \cap L^{2}}+\mathcal{E}_{N, \infty}(V(\sqrt{\gamma} t))\right),
\end{gathered}
$$

which imply (4.4) due to

$$
\|\tilde{B}(t)\| \leq C\|B(t / \sqrt{\gamma})\|, \quad\left\|\sigma_{+}(t)+\sigma_{-}(t)\right\| \leq C\left\|\rho_{+}(t / \sqrt{\gamma})+\rho_{-}(t / \sqrt{\gamma})\right\|
$$

and the fact that $\left[\rho_{ \pm}, u_{ \pm}, E, B\right]$ is equivalent with $\left[\sigma_{ \pm}, v_{ \pm}, \tilde{E}, \tilde{B}\right]$ up to a positive constant. This completes the proof of Lemma 4.2.

Now, the rest is to prove the uniform-in-time bound of $\mathcal{E}_{N, \infty}(V(t))$ which yields the timedecay rates of the Lyapunov functional $\mathcal{E}_{N}(V(t))$ and thus $\|V(t)\|_{N}^{2}$. In fact, by taking $\ell=\frac{3}{2}+\epsilon$ in (4.2) with $\epsilon>0$ small enough, one has

$$
\begin{aligned}
& (1+t)^{\frac{3}{2}+\epsilon} \mathcal{E}_{N}(V(t))+\lambda \int_{0}^{t}(1+s)^{\frac{3}{2}+\epsilon} \mathcal{D}_{N}(V(s)) d s \\
\leq & C \mathcal{E}_{N+2}\left(V_{0}\right)+C \int_{0}^{t}(1+s)^{\frac{1}{2}+\epsilon}\left(\|\tilde{B}\|^{2}+\left\|\left[\sigma_{+}+\sigma_{-}\right]\right\|^{2}\right) d s .
\end{aligned}
$$

Here, using (4.4) and the fact that $\mathcal{E}_{N, \infty}(V(t))$ is non-decreasing in $t$, it further holds that

$$
\begin{aligned}
& \int_{0}^{t}(1+s)^{\frac{1}{2}+\epsilon}\left(\|\tilde{B}\|^{2}+\left\|\left[\sigma_{+}+\sigma_{-}\right]\right\|^{2}\right) d s \\
\leq & C(1+t)^{\epsilon}\left(\mathcal{E}_{N, \infty}^{2}(V(t))+\left\|\left[\sigma_{ \pm 0}, v_{ \pm 0}\right]\right\|_{L^{1} \cap L^{2}}^{2}+\left\|\left[v_{ \pm 0}, \tilde{E}_{0}, \tilde{B}_{0}\right]\right\|_{L^{1} \cap \dot{H}^{2}}^{2}\right) .
\end{aligned}
$$

Therefore, it follows that

$$
\begin{aligned}
& (1+t)^{\frac{3}{2}+\epsilon} \mathcal{E}_{N}(V(t))+\lambda \int_{0}^{t}(1+s)^{\frac{3}{2}+\epsilon} \mathcal{D}_{N}(V(s)) d s \\
\leq & C \mathcal{E}_{N+2}\left(V_{0}\right)+C(1+t)^{\epsilon}\left(\mathcal{E}_{N, \infty}^{2}(V(t))+\left\|\left[\sigma_{ \pm 0}, v_{ \pm 0}\right]\right\|_{L^{1} \cap L^{2}}^{2}+\left\|\left[v_{ \pm 0}, \tilde{E}_{0}, \tilde{B}_{0}\right]\right\|_{L^{1} \cap \dot{H}^{2}}^{2}\right),
\end{aligned}
$$


which implies

$$
(1+t)^{\frac{3}{2}} \mathcal{E}_{N}(V(t)) \leq C\left(\mathcal{E}_{N+2}\left(V_{0}\right)+\mathcal{E}_{N, \infty}^{2}(V(t))+\left\|\left[\sigma_{ \pm 0}, v_{ \pm 0}\right]\right\|_{L^{1} \cap L^{2}}^{2}+\left\|\left[v_{ \pm 0}, \tilde{E}_{0}, \tilde{B}_{0}\right]\right\|_{L^{1} \cap \dot{H}^{2}}^{2}\right) .
$$

Thus, one has

$$
\mathcal{E}_{N, \infty}(V(t)) \leq C\left(\epsilon_{N+2}^{2}\left(V_{0}\right)+\mathcal{E}_{N, \infty}^{2}(V(t))\right)
$$

Here, recall the definition of $\epsilon_{N+2}\left(V_{0}\right)$. Since $\epsilon_{N+2}\left(V_{0}\right)>0$ is sufficiently small, $\mathcal{E}_{N, \infty}(V(t)) \leq$ $C \epsilon_{N+2}^{2}\left(V_{0}\right)$ holds true for any $t \geq 0$, which implies

$$
\|V(t)\|_{N} \leq C \mathcal{E}_{N}(V(t))^{1 / 2} \leq C \epsilon_{N+2}\left(V_{0}\right)(1+t)^{-\frac{3}{4}},
$$

for any $t \geq 0$. This proves (2.12) in Proposition 2.2.

4.2. Time rate for higher-order instant energy functional. This subsection turns to the proof of (2.13) in Proposition 2.2. We start with the high-order energy estimate by the following

Lemma 4.3. Let $V=\left[\sigma_{ \pm}, v_{ \pm}, \tilde{E}, \tilde{B}\right]$ be the solution to the Cauchy problem (2.2)-(2.3) with initial data $V_{0}=\left[\sigma_{ \pm 0}, v_{ \pm 0}, \tilde{E}_{0}, \tilde{B}_{0}\right]$ satisfying (2.4) in the sense of Proposition 2.1. Then if $\mathcal{E}_{N}\left(V_{0}\right)$ is sufficiently small, there are the higher-order instant energy functional $\mathcal{E}_{N}^{h}(\cdot)$ and the corresponding dissipation rate $\mathcal{D}_{N}^{h}(\cdot)$ such that

$$
\frac{d}{d t} \mathcal{E}_{N}^{h}(V(t))+\lambda \mathcal{D}_{N}^{h}(V(t)) \leq C\left\|\nabla\left(\sigma_{+}+\sigma_{-}\right)\right\|^{2}
$$

holds for any $t \geq 0$.

Proof. It can be done by modifying the proof of Theorem 2.1 a little. In fact, by letting the energy estimates made only on the higher-order derivatives, then corresponding to (2.16), (2.19), (2.25) and (2.27), it can be re-verified that

$$
\begin{aligned}
& \frac{1}{2} \frac{d}{d t}\|\nabla V\|_{N-1}^{2}+\frac{1}{\sqrt{\gamma}}\left\|\nabla\left[v_{+}, v_{-}\right]\right\|_{N-1}^{2} \leq C\|V\|_{N}\left\|\nabla\left[\sigma_{+}, \sigma_{-}, v_{+}, v_{-}\right]\right\|_{N-1}^{2}, \\
& \quad \frac{d}{d t} \sum_{1 \leq|\alpha| \leq N-1}\left(\left\langle\partial^{\alpha} v_{+}, \nabla \partial^{\alpha} \sigma_{+}\right\rangle+\left\langle\partial^{\alpha} v_{-}, \nabla \partial^{\alpha} \sigma_{-}\right\rangle\right)+\lambda\left\|\nabla^{2}\left[\sigma_{+}, \sigma_{-}\right]\right\|_{N-2}^{2} \\
& \leq C\left\|\nabla\left[v_{+}, v_{-}\right]\right\|_{N-1}^{2}+C\|V\|_{N}^{2}\left\|\nabla\left[\sigma_{+}, \sigma_{-}, v_{+}, v_{-}\right]\right\|_{N-1}^{2}, \\
& \quad \frac{d}{d t} \sum_{1 \leq|\alpha| \leq N-1}\left\langle\partial^{\alpha}\left(v_{+}-v_{-}\right), \partial^{\alpha} \tilde{E}\right\rangle+\lambda\|\nabla \tilde{E}\|_{N-2}^{2} \\
& \leq C\left\|\nabla\left[v_{+}, v_{-}\right]\right\|_{N-1}^{2}+C\left\|\nabla^{2}\left[\sigma_{+}, \sigma_{-}\right]\right\|_{N-2}^{2}+C\left\|\nabla\left[v_{+}, v_{-}\right]\right\|_{N-1}\left\|\nabla^{2} \tilde{B}\right\|_{N-3} \\
& \quad+C\|V\|_{N}^{2}\left\|\nabla\left[\sigma_{+}, \sigma_{-}, v_{+}, v_{-}\right]\right\|_{N-1}^{2}, \\
& \quad-\frac{d}{d t} \sum_{1 \leq|\alpha| \leq N-2}\left\langle\nabla \times \partial^{\alpha} \tilde{E}, \partial^{\alpha} \tilde{B}\right\rangle+\lambda\left\|\nabla^{2} \tilde{B}\right\|_{N-3}^{2} \\
& \left.\leq C \| \nabla^{2} \tilde{E}\right]\left\|_{N-3}^{2}+C\right\| \nabla\left[v_{+}, v_{-}\right]\left\|_{N-3}^{2}+C\right\| V\left\|_{N}^{2}\right\| \nabla\left[\sigma_{+}, \sigma_{-}, v_{+}, v_{-}\right] \|_{N-1}^{2} .
\end{aligned}
$$


Here, the details of proof are omitted for simplicity. Now, in the similar way as in (2.28), let us define

$$
\begin{aligned}
\mathcal{E}_{N}^{h}(V(t)) & =\|\nabla V\|_{N-1}^{2}+\kappa_{1} \sum_{1 \leq|\alpha| \leq N-1}\left(\left\langle\partial^{\alpha} v_{+}, \nabla \partial^{\alpha} \sigma_{+}\right\rangle+\left\langle\partial^{\alpha} v_{-}, \nabla \partial^{\alpha} \sigma_{-}\right\rangle\right) \\
& +\kappa_{2} \sum_{1 \leq|\alpha| \leq N-1}\left\langle\partial^{\alpha}\left(v_{+}-v_{-}\right), \nabla \partial^{\alpha} \tilde{E}\right\rangle-\kappa_{3} \sum_{1 \leq|\alpha| \leq N-2}\left\langle\nabla \times \partial^{\alpha} \tilde{E}, \partial^{\alpha} \tilde{B}\right\rangle .
\end{aligned}
$$

Similarly, one can choose $0<\kappa_{3} \ll \kappa_{2} \ll \kappa_{1} \ll 1$ with $\kappa_{2}^{3 / 2} \ll \kappa_{3}$, such that $\mathcal{E}_{N}^{h}(V(t)) \sim$ $\|\nabla V(t)\|_{N-1}^{2}$. Furthermore, the linear combination of the previously obtained four estimates with coefficients corresponding to (4.8) yields (4.7) with $\mathcal{D}_{N}^{h}(\cdot)$ defined in (2.8). This completes the proof of Lemma 4.3 .

By comparing (2.8) and (2.6) for the definitions of $\mathcal{E}_{N}^{h}(V(t))$ and $\mathcal{D}_{N}^{h}(V(t))$, it follows from (4.7) that

$$
\frac{d}{d t} \mathcal{E}_{N}^{h}(V(t))+\lambda \mathcal{E}_{N}^{h}(V(t)) \leq C\left(\|\nabla \tilde{B}\|^{2}+\left\|\nabla^{N}[\tilde{E}, \tilde{B}]\right\|^{2}+\left\|\nabla\left(\sigma_{+}+\sigma_{-}\right)\right\|^{2}\right),
$$

which implies

$$
\begin{aligned}
& \mathcal{E}_{N}^{h}(V(t)) \leq e^{-\lambda t} \mathcal{E}_{N}^{h}\left(V_{0}\right) \\
& +C \int_{0}^{t} e^{-\lambda(t-s)}\left(\|\nabla \tilde{B}(s)\|^{2}+\left\|\nabla^{N}[\tilde{E}, \tilde{B}](s)\right\|^{2}+\left\|\nabla\left(\sigma_{+}+\sigma_{-}\right)(s)\right\|^{2}\right) d s .
\end{aligned}
$$

To estimate the time integral term on the r.h.s. of the above inequality, one has

Lemma 4.4. Let $V=\left[\sigma_{ \pm}, v_{ \pm}, \tilde{E}, \tilde{B}\right]$ be the solution to the Cauchy problem (2.2)-(2.3) with initial data $V_{0}=\left[\sigma_{ \pm 0}, v_{ \pm 0}, \tilde{E}_{0}, \tilde{B}_{0}\right]$ satisfying (2.4) in the sense of Proposition 2.1. Then if $\epsilon_{N+6}\left(V_{0}\right)$ is sufficiently small, where $\epsilon_{N+6}\left(V_{0}\right)$ is defined in $(2.11)$, then

$$
\begin{aligned}
\|\nabla \tilde{B}(t)\|^{2}+\left\|\nabla^{N}[\tilde{E}(t), \tilde{B}(t)]\right\|^{2}+ & \left\|\nabla\left(\sigma_{+}(t)+\sigma_{-}(t)\right)\right\|^{2} \\
& \leq C \epsilon_{N+6}^{2}\left(V_{0}\right)(1+t)^{-\frac{5}{2}}
\end{aligned}
$$

holds for any $t \geq 0$.

For this time, suppose that the above lemma is true. Then by using (4.10) in (4.9), it is immediate to obtain

$$
\mathcal{E}_{N}^{h}(V(t)) \leq e^{-\lambda t} \mathcal{E}_{N}^{h}\left(V_{0}\right)+C \epsilon_{N+6}^{2}\left(V_{0}\right)(1+t)^{-\frac{5}{2}},
$$

which proves (2.13) in Proposition 2.2.

Proof of Lemma 4.4: Suppose that $\epsilon_{N+6}\left(V_{0}\right)>0$ is sufficiently small. Notice that, by the first part of Proposition 2.2,

$$
\|V(t)\|_{N+4} \leq C \epsilon_{N+6}\left(V_{0}\right)(1+t)^{-\frac{3}{4}}
$$

which further implies from $(2.1)$ that for $U=\left[\rho_{ \pm}, u_{ \pm}, E, B\right]$,

$$
\|U(t)\|_{N+4} \leq C \epsilon_{N+6}\left(V_{0}\right)(1+t)^{-\frac{3}{4}} .
$$

Similar to obtaining (4.5), one can apply the linear estimate (3.17) to the mild form (3.4) of the solution $U_{1}(t)$, and the linear estimate on $\rho_{2}$ to the mild form (3.6) of the solution $U_{2}(t)$ so that

$$
\begin{aligned}
& \|\nabla B(t)\| \leq C(1+t)^{-\frac{5}{4}}\left\|\left[u_{ \pm 0}, E_{0}, B_{0}\right]\right\|_{L^{1} \cap \dot{H}^{4}} \\
& +C \int_{0}^{t}(1+t-s)^{-\frac{5}{4}}\left\|\left[g_{2}^{+}(s)-g_{2}^{-}(s), g_{3}^{+}(s)-g_{3}^{-}(s)\right]\right\|_{L^{1} \cap \dot{H}^{4}} d s
\end{aligned}
$$




$$
\begin{aligned}
& \left\|\nabla^{N}[E(t), B(t)]\right\| \leq C(1+t)^{-\frac{5}{4}}\left\|\left[u_{ \pm 0}, E_{0}, B_{0}\right]\right\|_{L^{1} \cap \dot{H} N+3} \\
& +C \int_{0}^{t}(1+t-s)^{-\frac{5}{4}}\left\|\left[g_{2}^{+}(s)-g_{2}^{-}(s), g_{3}^{+}(s)-g_{3}^{-}(s)\right]\right\|_{L^{1} \cap \dot{H}^{N+3}} d s,
\end{aligned}
$$

and

$$
\begin{aligned}
\left\|\nabla\left(\rho_{+}(t)+\rho_{-}(t)\right)\right\| \leq C(1 & +t)^{-\frac{5}{4}}\left\|\left[\rho_{ \pm 0}, u_{ \pm 0}\right]\right\|_{L^{1}}+e^{-\lambda t}\left\|\nabla\left[\rho_{ \pm 0}, u_{ \pm 0}\right]\right\| \\
& +C \int_{0}^{t}(1+t-s)^{-\frac{5}{4}}\left\|\left[g_{1}^{+}(s)+g_{1}^{-}(s), g_{2}^{+}(s)+g_{2}^{-}(s)\right]\right\|_{L^{1}} d s \\
& +C \int_{0}^{t} e^{-\lambda(t-s)}\left\|\nabla\left[g_{1}^{+}(s)+g_{1}^{-}(s), g_{2}^{+}(s)+g_{2}^{-}(s)\right]\right\| d s .
\end{aligned}
$$

Recalling the definition (3.7), it is straightforward to verify

$$
\begin{gathered}
\left\|\left[g_{1}^{+}(t)+g_{1}^{-}(t), g_{2}^{+}(t)+g_{2}^{-}(t)\right]\right\|_{L^{1} \cap \dot{H}^{1}} \leq C\|U(t)\|_{4}^{2}, \\
\left\|\left[g_{2}^{+}(t)-g_{2}^{-}(t), g_{3}^{+}(t)-g_{3}^{-}(t)\right]\right\|_{L^{1} \cap \dot{H}^{4}} \leq C\|U(t)\|_{\max \{5, N\}}^{2}, \\
\left\|\left[g_{2}^{+}(t)-g_{2}^{-}(t), g_{3}^{+}(t)-g_{3}^{-}(t)\right]\right\|_{L^{1} \cap \dot{H}^{N+3}} \leq C\|U(t)\|_{N+4}^{2} .
\end{gathered}
$$

The above estimates together with (4.11) give

$$
\begin{aligned}
& \left\|\left[g_{1}^{+}(t)+g_{1}^{-}(t), g_{2}^{+}(t)+g_{2}^{-}(t)\right]\right\|_{L^{1} \cap \dot{H}^{1}}+\left\|\left[g_{2}^{+}(t)-g_{2}^{-}(t), g_{3}^{+}(t)-g_{3}^{-}(t)\right]\right\|_{L^{1} \cap \dot{H}^{4}} \\
& +\left\|\left[g_{2}^{+}(t)-g_{2}^{-}(t), g_{3}^{+}(t)-g_{3}^{-}(t)\right]\right\|_{L^{1} \cap \dot{H}^{N+3}} \leq C\|U(t)\|_{N+4}^{2} \leq C \epsilon_{N+6}^{2}\left(V_{0}\right)(1+t)^{-\frac{3}{2}} .
\end{aligned}
$$

Then it follows from (4.12), (4.13) and (4.14) that

$$
\begin{aligned}
\|\nabla B(t)\|+\left\|\nabla^{N}[E(t), B(t)]\right\|+ & \left\|\nabla\left[\rho_{+}(t)+\rho_{-}(t)\right]\right\| \\
& \leq C \epsilon_{N+6}\left(V_{0}\right)(1+t)^{-\frac{5}{4}},
\end{aligned}
$$

where the smallness of $\epsilon_{N+6}\left(V_{0}\right)$ was used. This implies (4.10) by the definition (2.1) of $\sigma_{ \pm}$, $\tilde{E}$ and $\tilde{B}$. The proof of Lemma 4.4 is complete.

4.3. Time rate in $L^{q}$. In this subsection we shall prove the time-decay rates in $L^{q}$ with $2 \leq q \leq \infty$ corresponding to (1.4)-(1.7) in Theorem 1.1 for solutions $U=\left[\rho_{ \pm}, u_{ \pm}, E, B\right]$ to the Cauchy problem (3.1)-(3.2). Throughout this subsection, we suppose that $\epsilon_{13}\left(V_{0}\right)>0$ is sufficiently small. In addition, for $N \geq 4$, Proposition 2.1 shows that if $\epsilon_{N+2}\left(V_{0}\right)$ is sufficiently small,

$$
\|U(t)\|_{N} \leq C \epsilon_{N+2}\left(V_{0}\right)(1+t)^{-\frac{3}{4}}
$$

and if $\epsilon_{N+6}\left(V_{0}\right)$ is sufficiently small,

$$
\|\nabla U(t)\|_{N-1} \leq C \epsilon_{N+6}\left(V_{0}\right)(1+t)^{-\frac{5}{4}} .
$$

Now, we begin with the estimates on $B,\left[u_{+}-u_{-}, E\right], u_{+}+u_{-}, \rho_{+}-\rho_{-}$and $\rho_{+}+\rho_{-}$in turn as follows.

Estimate on $\|B\|_{L^{q}}$. For $L^{2}$ rate, it is easy to see from (4.15) that

$$
\|B(t)\| \leq C \epsilon_{6}\left(V_{0}\right)(1+t)^{-\frac{3}{4}} .
$$

For $L^{\infty}$ rate, by applying the $L^{\infty}$ linear estimate on $B$ in (3.16) to the mild form (3.4), one has

$$
\begin{aligned}
\|B(t)\|_{\infty} \leq C(1+t)^{-\frac{3}{2}}\left\|\left[u_{ \pm 0}, E_{0}, B_{0}\right]\right\|_{L^{1} \cap \dot{H}^{5}} & \\
& +C \int_{0}^{t}(1+t-s)^{-\frac{3}{2}}\left\|\left[g_{2}^{+}(s)-g_{2}^{-}(s), g_{3}^{+}(s)-g_{3}^{-}(s)\right]\right\|_{L^{1} \cap \dot{H}^{5}} d s .
\end{aligned}
$$


Since by (4.15),

$$
\left\|\left[g_{2}^{+}(t)-g_{2}^{-}(t), g_{3}^{+}(t)-g_{3}^{-}(t)\right]\right\|_{L^{1} \cap \dot{H}^{5}} \leq C\|U(t)\|_{6}^{2} \leq C \epsilon_{8}^{2}\left(V_{0}\right)(1+t)^{-\frac{3}{2}}
$$

it follows that

$$
\|B(t)\|_{L^{\infty}} \leq C \epsilon_{8}\left(V_{0}\right)(1+t)^{-\frac{3}{2}}
$$

So, by $L^{2}-L^{\infty}$ interpolation,

$$
\|B(t)\|_{L^{q}} \leq C \epsilon_{8}\left(V_{0}\right)(1+t)^{-\frac{3}{2}+\frac{3}{2 q}}
$$

for $2 \leq q \leq \infty$.

Estimate on $\left\|\left[u_{+}-u_{-}, E\right]\right\|_{L^{q}}$. For $L^{2}$ rate, applying the $L^{2}$ linear estimate on $\left[u_{+}-u_{-}, E\right]$ in (3.15) to the mild form (3.4),

$$
\begin{gathered}
\left\|u_{+}(t)-u_{-}(t)\right\| \leq C(1+t)^{-\frac{5}{4}}\left(\left\|\rho_{ \pm 0}\right\|+\left\|\left[u_{ \pm 0}, E_{0}, B_{0}\right]\right\|_{L^{1} \cap \dot{H}^{2}}\right) \\
+C \int_{0}^{t}(1+t-s)^{-\frac{5}{4}}\left(\left\|g_{1}^{+}(s)-g_{1}^{-}(s)\right\|+\left\|\left[g_{2}^{+}(s)-g_{2}^{-}(s), g_{3}^{+}(s)-g_{3}^{-}(s)\right]\right\|_{L^{1} \cap \dot{H}^{2}}\right) d s,
\end{gathered}
$$

and

$$
\begin{aligned}
\|E(t)\| \leq & C(1+t)^{-\frac{5}{4}}\left\|\left[u_{ \pm 0}, E_{0}, B_{0}\right]\right\|_{L^{1} \cap \dot{H}^{3}} \\
& +C \int_{0}^{t}(1+t-s)^{-\frac{5}{4}}\left\|\left[g_{2}^{+}(s)-g_{2}^{-}(s), g_{3}^{+}(s)-g_{3}^{-}(s)\right]\right\|_{L^{1} \cap \dot{H}^{3}} d s .
\end{aligned}
$$

Since by (4.15),

$$
\begin{aligned}
&\left\|g_{1}^{+}(t)-g_{1}^{-}(t)\right\|+\left\|\left[g_{2}^{+}(t)-g_{2}^{-}(t), g_{3}^{+}(t)-g_{3}^{-}(t)\right]\right\|_{L^{1} \cap \dot{H}^{3}} \\
& \leq C\|U(t)\|_{4}^{2} \leq C \epsilon_{6}^{2}\left(V_{0}\right)(1+t)^{-\frac{3}{2}}
\end{aligned}
$$

it follows that

$$
\left\|u_{+}(t)-u_{-}(t)\right\|+\|E(t)\| \leq C \epsilon_{6}\left(V_{0}\right)(1+t)^{-\frac{5}{4}} .
$$

For $L^{\infty}$ rate, by applying the $L^{\infty}$ linear estimate on $u_{+}-u_{-}$and $E$ in (3.16) to (3.4), one has

$$
\begin{aligned}
& \left\|u_{+}(t)-u_{-}(t)\right\|_{\infty} \leq C(1+t)^{-2}\left(\left\|\rho_{ \pm 0}\right\|_{L^{2} \cap \dot{H}^{2}}+\left\|\left[u_{ \pm 0}, E_{0}, B_{0}\right]\right\|_{L^{1} \cap \dot{H}^{5}}\right) \\
& \quad+C \int_{0}^{t}(1+t-s)^{-2}\left(\left\|g_{1}^{+}(s)-g_{1}^{-}(s)\right\|_{L^{2} \cap \dot{H}^{2}}+\left\|\left[g_{2}^{+}(s)-g_{2}^{-}(s), g_{3}^{+}(s)-g_{3}^{-}(s)\right]\right\|_{L^{1} \cap \dot{H}^{5}}\right) d s
\end{aligned}
$$

and

$$
\begin{aligned}
\|E(t)\|_{\infty} \leq C(1+t)^{-2}\left\|\left[u_{ \pm 0}, E_{0}, B_{0}\right]\right\|_{L^{1} \cap \dot{H}^{6}} & \\
& +C \int_{0}^{t}(1+t-s)^{-2}\left\|\left[g_{2}^{+}(s)-g_{2}^{-}(s), g_{3}^{+}(s)-g_{3}^{-}(s)\right]\right\|_{L^{1} \cap \dot{H}^{6}} d s
\end{aligned}
$$

Since

$$
\begin{aligned}
& \left\|g_{1}^{+}(t)-g_{1}^{-}(t)\right\|_{L^{2} \cap \dot{H}^{2}}+\left\|\left[g_{2}^{+}(t)-g_{2}^{-}(t), g_{3}^{+}(t)-g_{3}^{-}(t)\right]\right\|_{\dot{H}^{5} \cap \dot{H}^{6}} \\
\leq & C\|\nabla U(t)\|_{6}^{2} \leq C \epsilon_{13}^{2}\left(V_{0}\right)(1+t)^{-\frac{5}{2}}
\end{aligned}
$$

and

$$
\begin{aligned}
\|\left[g_{2}^{+}(t)-g_{2}^{-}(t),\right. & \left.g_{3}^{+}(t)-g_{3}^{-}(t)\right]\left\|_{L^{1}} \leq C\right\| U(t) \|\left(\|\nabla U(t)\|+\left\|u_{ \pm}(t)\right\|\right) \\
& \leq C\left(\epsilon_{6}\left(V_{0}\right)(1+t)^{-\frac{3}{4}}\right)\left(\epsilon_{10}\left(V_{0}\right)(1+t)^{-\frac{5}{4}}\right) \leq C \epsilon_{10}^{2}\left(V_{0}\right)(1+t)^{-2},
\end{aligned}
$$

where (4.15), (4.16) and (4.18) were used, then, it follows that

$$
\|\left[u_{+}(t)-u_{-}(t), E(t) \|_{L^{\infty}} \leq C \epsilon_{13}\left(V_{0}\right)(1+t)^{-2} .\right.
$$


So, by $L^{2}-L^{\infty}$ interpolation,

$$
\|\left[u_{+}(t)-u_{-}(t), E(t) \|_{L^{q}} \leq C \epsilon_{13}\left(V_{0}\right)(1+t)^{-2+\frac{3}{2 q}},\right.
$$

for $2 \leq q \leq \infty$. A similar argument can be applied to the estimates on $\left\|u_{+}+u_{-}\right\|_{L^{p}}$. For $L^{2}$ and $L^{\infty}$ rate, applying the $L^{2}$ and $L^{\infty}$ linear estimate on $u_{+}+u_{-}$in (3.32) and (3.34) to the mild form (3.6), one has

$$
\left\|u_{+}(t)+u_{-}(t)\right\|_{L^{q}} \leq C \epsilon_{13}\left(V_{0}\right)(1+t)^{-2+\frac{3}{2 q}},
$$

for $2 \leq q \leq \infty$. Combining (4.19) and (4.20), we deduce

$$
\left\|u_{ \pm}(t)\right\|_{L^{q}} \leq C \epsilon_{13}\left(V_{0}\right)(1+t)^{-2+\frac{3}{2 q}}
$$

for $2 \leq q \leq \infty$.

Estimate on $\left\|\rho_{+}-\rho_{-}\right\|_{L^{q}}$. For $L^{2}$ rate, by applying the $L^{2}$ linear estimate on $\rho_{+}-\rho_{-}$in (3.15) to (3.4), one has

$$
\begin{aligned}
\left\|\rho_{+}(t)-\rho_{-}(t)\right\| \leq & C e^{-\frac{t}{2}}\left\|\left[\rho_{ \pm 0}, u_{ \pm 0}\right]\right\| \\
& +C \int_{0}^{t} e^{-\frac{t-s}{2}}\left\|\left[g_{1}^{+}(s)-g_{1}^{-}(s), g_{2}^{+}(s)-g_{2}^{-}(s)\right]\right\| d s .
\end{aligned}
$$

Due to

$$
\begin{aligned}
\left\|\left[g_{1}^{+}(t)-g_{1}^{-}(t), g_{2}^{+}(t)-g_{2}^{-}(t)\right]\right\| & \leq C\left(\|\nabla U(t)\|_{1}^{2}+\left\|u_{+}(t)+u_{-}(t)\right\| \cdot\|B(t)\|_{\infty}\right) \\
& \leq C \epsilon_{10}^{2}\left(V_{0}\right)(1+t)^{-\frac{5}{2}}
\end{aligned}
$$

where $(4.16),(4.17),(4.20)$ were used, then (4.21) gives the slower time-decay estimate

$$
\left\|\rho_{+}(t)-\rho_{-}(t)\right\| \leq C \epsilon_{10}\left(V_{0}\right)(1+t)^{-\frac{5}{2}} .
$$

Similarly for $\left\|\rho_{+}+\rho_{-}\right\|$, by using the $L^{2}$ linear estimate on $\rho_{+}+\rho_{-}$in (3.32) to (3.6), one has the slower time-decay estimate

$$
\left\|\rho_{+}(t)+\rho_{-}(t)\right\| \leq C \epsilon_{10}\left(V_{0}\right)(1+t)^{-\frac{3}{4}} .
$$

Then from (4.23) and (4.24) we have

$$
\left\|\rho_{ \pm}(t)\right\| \leq C \epsilon_{10}\left(V_{0}\right)(1+t)^{-\frac{3}{4}} .
$$

For $L^{\infty}$ rate, by applying the $L^{\infty}$ linear estimates on $\rho_{+}-\rho_{-}$in (3.16) to (3.4), one has

$$
\begin{aligned}
\left\|\rho_{+}(t)-\rho_{-}(t)\right\|_{L^{\infty}} \leq & C e^{-\frac{t}{2}}\left\|\left[\rho_{ \pm 0}, u_{ \pm 0}\right]\right\|_{L^{2} \cap \dot{H}^{2}} \\
& +\int_{0}^{t} e^{-\frac{t-s}{2}}\left\|\left[g_{1}^{+}(s)-g_{1}^{-}(s), g_{2}^{+}(s)-g_{2}^{-}(s)\right]\right\|_{L^{2} \cap \dot{H}^{2}} d s .
\end{aligned}
$$

Notice that one can check

$$
\left\|\left[g_{1}^{ \pm}(t), g_{2}^{ \pm}(t)\right]\right\|_{\dot{H}^{2}} \leq C\|\nabla U(t)\|_{4}\left(\left\|\rho_{ \pm}(t)\right\|+\left\|\left[u_{ \pm}(t), B(t)\right]\right\|_{L^{\infty}}+\left\|u_{ \pm}(t)\right\|\right) .
$$

The above inequality and (4.22) imply

$$
\left\|\left[g_{1}^{ \pm}(t), g_{2}^{ \pm}(t)\right]\right\|_{\dot{H}^{2} \cap L^{2}} \leq C \epsilon_{13}\left(V_{0}\right)(1+t)^{-2},
$$

which from (4.25), further gives

$$
\left\|\left[\rho_{+}(t)-\rho_{-}(t)\right]\right\|_{L^{\infty}} \leq C \epsilon_{13}\left(V_{0}\right)(1+t)^{-2} .
$$

So, by $L^{2}-L^{\infty}$ interpolation,

$$
\left\|\left[\rho_{+}(t)-\rho_{-}(t)\right]\right\|_{L^{q}} \leq C \epsilon_{13}\left(V_{0}\right)(1+t)^{-2-\frac{1}{q}},
$$

for $2 \leq q \leq \infty$. 
For $\left\|\rho_{+}+\rho_{-}\right\|_{L^{\infty}}$, by using the $L^{\infty}$ linear estimate on $\rho_{+}+\rho_{-}$in (3.34) to (3.6), one has the slower time-decay estimate

$$
\left\|\rho_{+}(t)+\rho_{-}(t)\right\|_{L^{\infty}} \leq C \epsilon_{13}\left(V_{0}\right)(1+t)^{-\frac{3}{2}} .
$$

Combining (4.27) and (4.24), we deduce that

$$
\left\|\rho_{+}(t)+\rho_{-}(t)\right\|_{L^{q}} \leq C \epsilon_{13}\left(V_{0}\right)(1+t)^{-\frac{3}{2}+\frac{3}{2 q}},
$$

for $2 \leq q \leq \infty$.

Thus, (4.17), (4.19), (4.20), (4.26), (4.28) give (1.7), (1.6), (1.4), and (1.5) respectively. This completes the proof of Theorem 1.1.

\section{Appendix}

In this appendix, as a byproduct of Theorem 3.1 and Corollary 3.1, we shall improve the results in [18] about the time-decay rates of solutions to the following Cauchy problem on the damped Euler system:

$$
\left\{\begin{array}{l}
\partial_{t} u+\nabla \cdot v=-v \cdot \nabla u-\frac{\gamma-1}{2} u \nabla \cdot v, \\
\partial_{t} v+\nabla u+a v=-v \cdot \nabla v-\frac{\gamma-1}{2} u \nabla u,
\end{array}\right.
$$

with initial data

$$
\left.(u, v)\right|_{t=0}=\left(u_{0}, v_{0}\right),
$$

where $a>0$ is a constant. Here, we have used the notion $[u, v]$ instead of $\left[\rho_{2}, u_{2}\right]$ for convenience of comparison with [18]. In fact, from Corollary 3.1, we notice that the decay rates $(3.32),(3.33)$ and (3.34) for the linearized system of (5.1) are much better than those in Lemma 6.1 of [18]. In order to obtain the same rates in the nonlinear case, we need the energy inequality of solutions under smallness of initial data as in (4.1).

Lemma 5.1. For the damped Euler system (5.1), the solution $[u, v]$ to the Cauchy problem of (5.1) with initial data $\left[u_{0}, v_{0}\right]$ satisfying $\left\|\left[u_{0}, v_{0}\right]\right\|_{H^{3}} \leq \delta$ for $\delta>0$ small enough has the following proposition

$$
\frac{d}{d t}\|[u, v]\|_{H^{3}}^{2}+\lambda\left(\|\nabla u\|_{H^{2}}^{2}+\|v\|_{H^{3}}^{2}\right) \leq 0
$$

for any $t>0$.

Proof. For each multi-index $|\alpha| \leq 3$, from the first two equations of (5.1), energy estimates on $\partial^{\alpha} u$ and $\partial^{\alpha} v$ give

$$
\frac{1}{2} \frac{d}{d t}\left\|\partial^{\alpha}[u, v]\right\|^{2}+a\left\|\partial^{\alpha} v\right\|^{2}=-\sum_{\beta<\alpha} C_{\beta}^{\alpha} I_{\alpha, \beta}(t)+I_{1}(t)
$$

with

$$
\begin{aligned}
I_{\alpha, \beta}(t)= & \left\langle\partial^{\alpha-\beta} v \cdot \nabla \partial^{\beta} u, \partial^{\alpha} u\right\rangle+\frac{\gamma-1}{2}\left\langle\partial^{\alpha-\beta} u \nabla \cdot \partial^{\beta} v, \partial^{\alpha} u\right\rangle \\
& +\frac{\gamma-1}{2}\left\langle\partial^{\alpha-\beta} u \nabla \partial^{\beta} u, \partial^{\alpha} v\right\rangle+\left\langle\partial^{\alpha-\beta} v \cdot \nabla \partial^{\beta} v, \partial^{\alpha} v\right\rangle,
\end{aligned}
$$

and

$$
I_{1}(t)=\frac{1}{2}\left\langle\nabla \cdot v,\left|\partial^{\alpha} u\right|^{2}+\left|\partial^{\alpha} v\right|^{2}\right\rangle+\frac{\gamma-1}{2}\left\langle\partial^{\alpha} v \cdot \nabla u, \partial^{\alpha} u\right\rangle,
$$


where integration by parts was used. In a similar way as in the proof of Theorem 2.1, we can bound $\left|I_{1}(t)\right|+\left|I_{\alpha, \beta}(t)\right|$ by

$$
\left|I_{1}(t)\right|+\left|I_{\alpha, \beta}(t)\right| \leq C\|[u, v]\|_{H^{1}}\|\nabla[u, v]\|_{H^{2}}^{2} .
$$

Taking summation over $|\alpha| \leq 3$, we deduce

$$
\frac{1}{2} \frac{d}{d t}\|[u, v]\|_{H^{3}}^{2}+a\|v\|_{H^{3}}^{2} \leq C\|[u, v]\|_{H^{3}}\|\nabla[u, v]\|_{H^{2}}^{2} .
$$

Furthermore, to include the estimate on $\left\|\nabla \partial^{\alpha} u\right\|^{2}$ when $|\alpha| \leq 2$, applying $\partial^{\alpha}$ to $(5.1)_{2}$ taking integrations in $x$ and then using integration by parts, and also replacing $\partial_{t} u$ from $(5.1)_{1}$ gives

$$
\begin{aligned}
& \frac{d}{d t}\left\langle\partial^{\alpha} v, \nabla \partial^{\alpha} u\right\rangle+\left\|\nabla \partial^{\alpha} u\right\|^{2} \\
= & -a\left\langle\partial^{\alpha} v, \nabla \partial^{\alpha} u\right\rangle+\left\|\nabla \cdot \partial^{\alpha} v\right\|^{2}+\left\langle\partial^{\alpha}\left(v \cdot \nabla u+\frac{\gamma-1}{2} u \nabla \cdot v\right), \nabla \cdot \partial^{\alpha} v\right\rangle \\
& -\left\langle\partial^{\alpha}\left(v \cdot \nabla v+\frac{\gamma-1}{2} u \nabla u\right), \nabla \partial^{\alpha} u\right\rangle .
\end{aligned}
$$

Then, it follows from Cauchy-Schwarz inequality and taking summation over $|\alpha| \leq 2$ that

$$
\frac{d}{d t} \sum_{|\alpha| \leq 2}\left\langle\partial^{\alpha} v, \nabla \partial^{\alpha} u\right\rangle+\lambda\|\nabla u\|_{H^{2}}^{2} \leq C\|v\|_{H^{3}}^{2}+\|[u, v]\|_{H^{3}}^{2}\|\nabla[u, v]\|_{H^{2}}^{2} .
$$

Combining (5.4) and (5.5), similar to Lemma 4.1, (5.3) follows due to smallness of initial data. This completes the proof of Lemma 5.1.

Proposition 5.1. Let $[u, v]$ be the solution to the Cauchy problem of (5.1)-(5.2) with initial data satisfying $\left\|\left[u_{0}, v_{0}\right]\right\|_{H^{3}}+\left\|\left[u_{0}, v_{0}\right]\right\|_{L^{1}} \leq \delta$ for $\delta>0$ small enough. Then, one has the following time-decay estimates:

$$
\begin{array}{cc}
\|u(t)\| \leq C(1+t)^{-\frac{3}{4}}, \quad\|\nabla u(t)\| \leq C(1+t)^{-\frac{5}{4}}, \quad\|u(t)\|_{L^{\infty}} \leq C(1+t)^{-\frac{3}{2}}, \\
\|v(t)\| \leq C(1+t)^{-\frac{5}{4}}, \quad\|\nabla v(t)\| \leq C(1+t)^{-\frac{7}{4}}, \quad\|v(t)\|_{L^{\infty}} \leq C(1+t)^{-2},
\end{array}
$$

for any $t \geq 0$, Furthermore, $\|[u, v]\|_{H^{3}} \leq C(1+t)^{-\frac{3}{4}}$.

Proof. Define the time-weighted sup-norms:

$$
\begin{gathered}
L_{0}(t)=\sup _{0 \leq s \leq t}\left\{(1+s)^{\frac{3}{4}}\|u(\cdot, s)\|+(1+s)^{\frac{5}{4}}\|v(\cdot, s)\|\right\}, \\
L_{1}(t)=\sup _{0 \leq s \leq t}\left\{(1+s)^{\frac{5}{4}}\|\nabla u(\cdot, s)\|+(1+s)^{\frac{7}{4}}\|\nabla v(\cdot, s)\|\right\}, \\
L_{\infty}(t)=\sup _{0 \leq s \leq t}\left\{(1+s)^{\frac{3}{2}}\|u(\cdot, s)\|_{L^{\infty}}+(1+s)^{2}\|v(\cdot, s)\|_{L^{\infty}}\right\}, \\
\mathcal{E}(t)=\sup _{0 \leq s \leq t}\left\{(1+s)^{\frac{3}{4}}\|[u, v](\cdot, s)\|_{H^{3}}\right\} .
\end{gathered}
$$

In the following, we will prove the bound of $L_{0}(t), \mathcal{E}(t), L_{1}(t)$, and $L_{\infty}(t)$ by four steps.

\section{Step 1. The bound of $L_{0}(t)$}

It follows from Theorem 3.1 and Duhamel principle, we have

$$
\|v(t)\| \leq C(1+t)^{-\frac{5}{4}}\left\|\left[u_{0}, v_{0}\right]\right\|_{L^{1}}+C e^{-\lambda t}\left\|\left[u_{0}, v_{0}\right]\right\|
$$

$$
+\int_{0}^{t}(1+t-s)^{-\frac{5}{4}}\|G(U, \nabla U)(\cdot, s)\|_{L^{1}} d s+\int_{0}^{t} e^{-\lambda(t-s)}\|G(U, \nabla U)(\cdot, s)\| d s .
$$


By some direct calculations, we have the following estimates:

$$
\begin{gathered}
\|G(U, \nabla U)(\cdot, s)\|_{L^{1}} \leq C\|U(\cdot, s)\|\|\nabla U(\cdot, s)\| \leq C L_{0}(t) L_{1}(t)(1+s)^{-\frac{9}{4}} \\
\|G(U, \nabla U)(\cdot, s)\|_{L^{2}} \leq C\|U(\cdot, s)\|_{L^{\infty}}\|\nabla U(\cdot, s)\| \leq C L_{\infty}(t) L_{1}(t)(1+s)^{-\frac{11}{4}} .
\end{gathered}
$$

The above two estimates (5.7)-(5.8) together with (5.6) give

$$
\|v(t)\| \leq C(1+t)^{-\frac{5}{4}}\left(\delta+L_{0}(t) L_{1}(t)+L_{1}(t) L_{\infty}(t)\right) .
$$

Similarly, we have

$$
\|u(t)\| \leq C(1+t)^{-\frac{3}{4}}\left(\delta+L_{0}(t) L_{1}(t)+L_{1}(t) L_{\infty}(t)\right) .
$$

Therefore, we have

$$
L_{0}(t) \leq C\left(\delta+L_{0}(t) L_{1}(t)+L_{1}(t) L_{\infty}(t)\right) .
$$

\section{Step 2. The bound of $\mathcal{E}(t)$}

From Lemma 5.1, (5.3) implies a Lyapunov-type inequality

$$
\frac{d}{d t}\|[u, v]\|_{H^{3}}^{2}+\lambda\|[u, v]\|_{H^{3}}^{2} \leq C\|u\|^{2} .
$$

It is immediate to obtain

$$
\begin{aligned}
\|[u, v]\|_{H^{3}}^{2} & \leq C e^{-\lambda t}\left\|\left[u_{0}, v_{0}\right]\right\|+\int_{0}^{t} e^{-\lambda(t-s)}\|u(s)\|^{2} d s \\
& \leq C(1+t)^{-\frac{3}{2}}\left(\delta+L_{0}(t) L_{1}(t)+L_{1}(t) L_{\infty}(t)\right),
\end{aligned}
$$

where we have used the estimate of $\|u\|$ in (4.18). (5.10) implies that $\mathcal{E}(t)$ is bounded in the following form

$$
\mathcal{E}(t) \leq C\left(\delta+L_{0}(t) L_{1}(t)+L_{1}(t) L_{\infty}(t)\right)
$$

Step 3. The bound of $L_{1}(t)$

It follows from Theorem 3.1 and Duhamel's principle, we have

$\|\nabla v(t)\| \leq C(1+t)^{-\frac{7}{4}}\left\|\left[u_{0}, v_{0}\right]\right\|_{L^{1}}+C e^{-\lambda t}\left\|\nabla\left[u_{0}, v_{0}\right]\right\|$

$$
+\int_{0}^{t}(1+t-s)^{-\frac{7}{4}}\|G(U, \nabla U)(\cdot, s)\|_{L^{1}}(s) d s+\int_{0}^{t} e^{-\lambda(t-s)}\|\nabla G(U, \nabla U)(\cdot, s)\| d s .
$$

Here, we only to estimate $\|\nabla G(U, \nabla U)\|$ as follows:

$$
\begin{aligned}
\|\nabla G(U, \nabla U)(\cdot, s)\| & \leq C\|U(\cdot, s)\|_{L^{\infty}}\left\|\nabla^{2} U(\cdot, s)\right\|+\|\nabla U(\cdot, s)\|_{L^{4}}^{2} \\
& \leq C\|U(\cdot, s)\|_{L^{\infty}}\left\|\nabla^{2} U(\cdot, s)\right\| \\
& \leq C L_{\infty}(t) \mathcal{E}(t)(1+s)^{-\frac{9}{4}}
\end{aligned}
$$

Plugging the above estimate and (5.7) into (5.12), we have

$$
\|\nabla v(t)\| \leq C(1+t)^{-\frac{7}{4}}\left(\delta+L_{0}(t) L_{1}(t)+L_{\infty}(t) \mathcal{E}(t)\right) .
$$

Similarly, we have

$$
\begin{aligned}
\|\nabla u(t)\| \leq & C(1+t)^{-\frac{5}{4}}\left\|\left[u_{0}, v_{0}\right]\right\|_{L^{1}}+C e^{-\lambda t}\left\|\nabla\left[u_{0}, v_{0}\right]\right\| \\
& +\int_{0}^{t}(1+t-s)^{-\frac{5}{4}}\|G(U, \nabla U)(\cdot, s)\|_{L^{1}}(s) d s+\int_{0}^{t} e^{-\lambda(t-s)}\|\nabla G(U, \nabla U)(\cdot, s)\| d s \\
(5.14) \leq & C(1+t)^{-\frac{5}{4}}\left(\delta+L_{0}(t) L_{1}(t)+L_{\infty}(t) \mathcal{E}(t)\right) .
\end{aligned}
$$


Combing (5.13) and (5.14), we have

$$
L_{1}(t) \leq C\left(\delta+L_{0}(t) L_{1}(t)+L_{1}(t) \mathcal{E}(t)\right)
$$

\section{Step 4. The bound of $L_{\infty}(t)$}

It follows from Theorem 3.1 and Duhamel principle, we have

$$
\begin{aligned}
\|v(t)\|_{L^{\infty}} \leq & C(1+t)^{-2}\left\|\left[u_{0}, v_{0}\right]\right\|_{L^{1}}+C e^{-\lambda t}\left\|\nabla^{2}\left[u_{0}, v_{0}\right]\right\| \\
& +\int_{0}^{t}(1+t-s)^{-2}\|G(U, \nabla U)(\cdot, s)\|_{L^{1}}(s) d s \\
& +\int_{0}^{t} e^{-\lambda(t-s)}\left\|\nabla^{2} G(U, \nabla U)(\cdot, s)\right\| d s .
\end{aligned}
$$

The rest is to obtain the estimate of $\left\|\nabla^{2} G(U, \nabla U)\right\|$,

$$
\begin{aligned}
\left\|\nabla^{2} G(U, \nabla U)(\cdot, s)\right\| & \leq C\|U(\cdot, s)\|_{L^{\infty}}\left\|\nabla^{3} U(\cdot, s)\right\|+C\left\|\nabla^{2} U \nabla U\right\| \\
& \leq C\|U(\cdot, s)\|_{L^{\infty}}\left\|\nabla^{3} U(\cdot, s)\right\| \\
& \leq C L_{\infty}(t) \mathcal{E}(t)(1+s)^{-\frac{9}{4}}
\end{aligned}
$$

which implies

$$
\|v(t)\|_{L^{\infty}} \leq C(1+t)^{-2}\left(\delta+L_{0}(t) L_{1}(t)+L_{\infty}(t) \mathcal{E}(t)\right) .
$$

The similar argument for $\|u(t)\|_{L^{\infty}}$ with decay rate $(1+t)^{-\frac{3}{2}}$, then we have

$$
L_{\infty}(t) \leq C\left(\delta+L_{0}(t) L_{1}(t)+\mathcal{E}(t) L_{\infty}(t)\right)
$$

Now, define $Q(t)=L_{\infty}(t)+L_{0}(t)+L_{1}(t)+\mathcal{E}(t)$. The summation of (5.9), (5.11), (5.15) and (5.16) implies that

$$
Q(t) \leq C\left(\delta+Q(t)^{2}\right)
$$

Since $\delta>0$ is small enough, $Q(t) \leq C \delta$ holds true for any $t \geq 0$, which implies $L_{0}(t), L_{1}(t)$, $\mathcal{E}(t)$ and $L_{\infty}(t)$ is bounded for all time. Then the proof of Proposition 5.1 is complete.

Remark 5.1. The rates shown in Proposition 5.1 are better than Theorem 6.1 in [18] in the sense that the momentum component has an extra time-decay $(1+t)^{-\frac{1}{2}}$ in $L^{2}$. In this sense, the appendix here can be viewed as a generalization of [18] with respect to the time-decay rates.

Acknowledgements: RJD was partially supported by the Direct Grant 2010/2011 in CUHK. QQL and CJZ were supported by the National Natural Science Foundation of China \#11071093, the PhD specialized grant of the Ministry of Education of China \#20100144110001, and the Special Fund for Basic Scientific Research of Central Colleges \#CCNU10C01001. The authors would like to thank the referees for useful comments to improve the presentation of this paper. 


\section{REFERENCES}

[1] C. Besse, J. Claudel, P. Degond, et al., A model hierarchy for ionospheric plasma modeling, Math. Models Methods Appl. Sci., 14 (2004), 393-415.

[2] Y. Brenier, N. Mauser and M. Puel, Incompressible Euler and e-MHD as scaling limits of the VlasovMaxwell system, Comm. Math. Sci., 1 (2003), 437-447.

[3] D. Chae and E. Tadmor, On the finite time blow-up of the Euler-Poisson equations in $R^{n}$, Comm. Math. Sci., 6 (2008), 785-789.

[4] G.Q. Chen, J.W. Jerome and D.H. Wang, Compressible Euler-Maxwell equations, Transpot Theory Statist. Phys., 29 (2000), 311-331.

[5] Y. Deng, T.P. Liu, T. Yang and Z.A. Yao, Solutions of Euler-Poisson equations for gaseous stars, Arch. Ration. Mech. Anal., 164 (2002), 261-285.

[6] R.J. Duan, Global smooth flows for the compressible Eluer-Maxwell system: Relaxation case, J. Hyperbolic Differential Equations, 8 (2011), no. 2, 375-413.

[7] R.J. Duan, S. Ukai and T. Yang, A combination of energy method and spectral analysis for study of equations of gas motion, Front. Math. China, 4(2009), 253-282.

[8] Y. Guo, Smooth irrotational flows in the large to the Euler-Poisson system in $\mathbb{R}^{3+1}$, Comm. Math. Phys., 195 (1998), 249-265.

[9] Y. Guo, B. Pausader, Global smooth ion dynamics in the Euler-Poisson system, Comm. Math. Phys., 303 (2011), no. 1, 89-125.

[10] D. Hoff and K. Zumbrun, Multi-dimensional diffusion waves for the Navier-Stokes equations of compressible flow, Indiana Univ. Math. J., 44 (1995), 603-676.

[11] J. W. Jerome, The Cauchy problem for compressible hydrodynamic-Maxwell systems: a local theory for smooth solutions, Differential Integral Equations, 16 (2003), 1345-1368.

[12] S. Kawashima, Systems of a hyperbolic-parabolic composite type, with applications to the equations of magnetohydrodynamics, Ph.D. Thesis, Kyoto Univ., 1983.

[13] T. Kato, The Cauchy problem for quasi-linear symmetric hyperbolic systems, Arch. Rational Mech. Anal., 58 (1975), 181-205.

[14] T. Luo, R. Natalini and Z.P. Xin, Large time behavior of the solutions to a hydrodynamic model for semiconductors, SIAM J. Appl. Math., 59 (1999), 810-830.

[15] T. Luo and J. Smoller, Existence and non-linear stability of rotating star solutions of the compressible Euler-Poisson equations, Arch. Ration. Mech. Anal., 191 (2009), 447-496.

[16] Y.J. Peng and S. Wang, Convergence of compressible Euler-Maxwell equations to incompressible Euler equations, Comm. Partial Differential Equations, 33 (2008), 349-376.

[17] H. Rishbeth and O.K. Garriott, Introduction to Ionospheric Physics. Academic Press, 1969.

[18] T.C. Sideris, B. Thomases and D.H. Wang, Long time behavior of solutions to the 3D compressible Euler equations with damping, Comm. Partial Differential Equations, 28 (2003), 795-816.

[19] E.M. Stein, Singular Integrals and Differentiability Properties of Functions, Princeton Mathematical Series, No. 30 Princeton University Press, Princeton, N.J. 1970 xiv+290 pp.

[20] M.E. Taylor, Partial Differential Equations, I. Basic Theory, Springer, New York, 1996.

[21] Y. Ueda and S. Kawashima, Decay property of regularity-loss type for the Euler-Maxwell system, preprint.

[22] Y. Ueda, S. Wang and S. Kawashima, Dissipative structure of the regularity-loss type and time asymptotic decay of solutions for the Euler-Maxwell system, preprint.

[23] C. Villani, Hypocoercivity, Memoirs Amer. Math. Soc., 202 (2009).

(RJD) Department of Mathematics, The Chinese University of Hong Kong, Shatin, Hong KONG

E-mail address: rjduan@math.cuhk.edu.hk

(QQL) The Hubei Key Laboratory of Mathematical Physics, School of Mathematics and Statistics, Central China Normal University, Wuhan, 430079, P. R. China

E-mail address: shuxueliuqingqing@126.com

(CJZ) The Hubei Key Laboratory of Mathematical Physics, School of Mathematics and Statistics, Central China Normal University, Wuhan, 430079, P. R. China

E-mail address: cjzhu@mail.ccnu.edu.cn 